ARTICLE

\title{
LSD1 inhibition sustains T cell invigoration with a durable response to PD-1 blockade
}

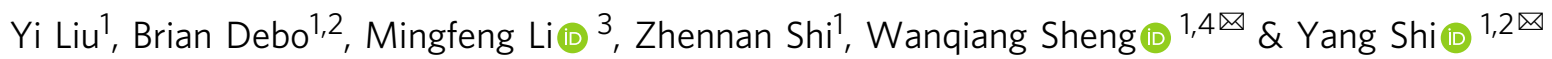

Exhausted $\mathrm{CD}^{+} \mathrm{T}$ cells are key targets of immune checkpoint blockade therapy and their ineffective reinvigoration limits the durable benefit in some cancer patients. Here, we demonstrate that histone demethylase LSD1 acts to enforce an epigenetic program in progenitor exhausted $\mathrm{CD}^{+}{ }^{+} \mathrm{T}$ cells to antagonize the TCF1-mediated progenitor maintenance and to promote terminal differentiation. Consequently, genetic perturbation or small molecules targeting LSD1 increases the persistence of the progenitor exhausted $\mathrm{CD} 8^{+} \mathrm{T}$ cells, which provide a sustained source for the proliferative conversion to numerically larger terminally exhausted T cells with tumor-killing cytotoxicity, thereby leading to effective and durable responses to anti-PD1 therapy. Collectively, our findings provide important insights into epigenetic mechanisms that regulate $T$ cell exhaustion and have important implications for durable immunotherapy.

\footnotetext{
${ }^{1}$ Division of Newborn Medicine and Epigenetics Program, Boston Children's Hospital, Harvard Medical School, Boston, MA 02115, USA. ${ }^{2}$ Ludwig Institute for Cancer Research, University of Oxford, Oxford OX3 7DQ, UK. ${ }^{3}$ Department of Neuroscience and Kavli Institute for Neuroscience, Yale School of Medicine, New Haven, CT 06510, USA. ${ }^{4}$ Institute of Immunology, and Department of Respiratory Disease of The First Affiliated Hospital, Zhejiang University School of

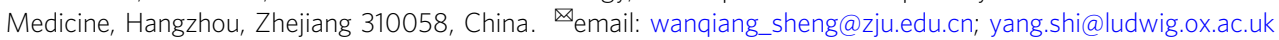


mmune checkpoint blockade, exemplified by using PD-1 blocking antibodies, has shown remarkable clinical success ${ }^{1,2}$. However, a majority of cancer patients have not benefited from this therapy to date. Notably, some cancer patients who initially respond to anti-PD-1 therapy develop tumor progression after a period of time in spite of continuous treatment ${ }^{1,3}$, highlighting the importance of improving $\mathrm{T}$ cell response to PD-1 blockade. The prolonged stimulation of $\mathrm{T}$ cell receptor (TCR) by cognate antigens drives $\mathrm{CD}^{+} \mathrm{T}$ cell exhaustion, which is maintained by the interaction between the high-level of PD-1 in T cells and PD-L1 in tumors $2,4,5$. The use of PD-1 blocking antibodies has been shown to abrogate this inhibitory effect and reinvigorate the exhausted $\mathrm{T}$ cells ${ }^{2,4}$. However, the molecular mechanisms leading to $\mathrm{T}$ cell exhaustion and to what extent exhausted $\mathrm{T}$ cells can be reinvigorated remain incompletely understood. Recently, in chronic viral infections and cancer ${ }^{6-8}$, exhausted $\mathrm{CD} 8^{+} \mathrm{T}$ cells have been defined to include at least two distinct subsets - a progenitor subset expressing an intermediate level of $\mathrm{PD}-1$ and the transcription factor TCF1 $\left(\mathrm{PD}-1^{\text {int }} \mathrm{TCF} 1^{+}\right)$, and a more differentiated subset lacking TCF1 while expressing a high level of PD-1 (PD-1 ${ }^{\text {hiTCF1-) }}$ that contains the terminally exhausted cells ${ }^{9-13}$. The progenitor exhausted $\mathrm{CD}^{+} \mathrm{T}$ cells retain higher proliferation capacity and better ability to produce cytokines, and can maintain self-renewal while continuously giving rise to numerically more differentiated cells that have stronger cytotoxicity, but are increasingly prone to apoptosis ${ }^{2,4}$. In line with these properties, the progenitor exhausted $\mathrm{CD}^{+} \mathrm{T}$ cells have been reported as the prominent determinant of effective responses to PD-1 blockade $e^{6,8,9,13}$. Indeed, in melanoma patients receiving anti-PD-1 treatment, the frequency of intratumoral $\mathrm{TCF}^{+}{ }^{+}$progenitor $\mathrm{CD} 8{ }^{+} \mathrm{T}$ cells is correlated with positive outcomes $^{7}$. Since proliferation of the progenitor exhausted CD8 ${ }^{+}$ $\mathrm{T}$ cells in response to TCR stimulation and PD-1 blockade progressively leads to their conversion to terminally exhausted phenotype $^{2}$, approaches to maintain or expand the progenitor subset of exhausted $\mathrm{CD}^{+} \mathrm{T}$ cells may help sustain anti-tumor response induced by anti-PD-1 therapy. In support of this hypothesis, the duration of response in melanoma patients who respond to anti-PD-1 treatment is positively correlated with the frequency of progenitor exhausted $\mathrm{CD}^{+}{ }^{+} \mathrm{T}$ cells ${ }^{6}$. Thus, understanding the mechanisms that control progenitor exhausted $\mathrm{CD}^{+} \mathrm{T}$ cell generation, maintenance, and differentiation to a terminal exhaustion state is of particular importance for predicting tumor response to anti-PD-1 treatment and for enhancing the treatment efficacy.

Massive changes occur in chromatin landscape when $\mathrm{CD} 8^{+}$ $\mathrm{T}$ cells become exhausted in chronic viral infections and cancer6,14-17. Chromatin modifications have the potential to efficiently and stably silence relevant genes to create a barrier, which prevents the reinvigoration of exhausted $\mathrm{CD}^{+} \mathrm{T}$ cells in response to $\mathrm{PD}-1$ blockade. Indeed, the de novo DNA methyltransferase, DNMT3A, has been reported to mediate DNA methylation and transcriptional silencing of both cytotoxicityand self-renewal-related genes in exhausted $\mathrm{CD} 8^{+} \mathrm{T}$ cells, and the methylation status remains largely stable even upon anti-PD-L1 treatment $^{18}$. In addition, histone methyltransferase EZH2 has also been reported to regulate $\mathrm{CD} 8^{+} \mathrm{T}$ cell differentiation and function in viral infections or cancer ${ }^{19-22}$, but the impact of perturbing $\mathrm{T}$ cell-intrinsic $\mathrm{EZH} 2$ on $\mathrm{T}$ cell response to $\mathrm{PD}-1$ blockade remains unclear. While a number of transcription factors, including TOX, T-bet, and Nr4A, have been discovered to regulate $\mathrm{T}$ cell exhaustion ${ }^{23-29}$, chromatin regulatory mechanisms in $\mathrm{T}$ cell exhaustion have not been fully explored. The identification and characterization of new epigenetic regulators in $\mathrm{T}$ cell exhaustion could uncover potential druggable targets for therapeutic intervention to potentiate the effectiveness and sustainability of PD-1 blockade therapy.
In this study, we identify histone demethylase LSD1 as an important modulator of T cell exhaustion in cancer. LSD1 loss in T cells expands the pool size of the progenitor subset of exhausted $\mathrm{CD}^{+} \mathrm{T}$ cells in a variety of mouse tumor models. Mechanistically, we demonstrate that LSD1 physically interacts with the long isoform of TCF1 and antagonizes its transcriptional activity. Consequently, LSD1 inhibition augments the transcriptional network controlled by TCF1 essential for maintaining the progenitor phenotype. In response to PD-1 blocking antibodies, the increased pool of progenitor exhausted $\mathrm{CD} 8^{+} \mathrm{T}$ cells caused by LSD1 inhibition provides a sustained source for the conversion to more differentiated $\mathrm{T}$ cells with stronger tumor-killing cytotoxicity, which enables a long-lasting response to anti-PD-1 treatment.

\section{Results}

LSD1 modulates $\mathrm{T}$ cell immunity depending on tumor context. To investigate the role of LSD1 in T cell immunity in the cancer context, we generated T cell-specific Lsd1 knockout (Cd4-Cre ${ }^{+}$ $\left.L s d 1^{f / f}\right)$ mice, which showed largely unaltered $\alpha \beta \mathrm{T}$ cell development (Supplementary Fig. 1a-c) and displayed an increased central memory phenotype of $\mathrm{T}$ cells in peripheral lymphoid organs (Supplementary Fig. 1d-1). We subcutaneously inoculated syngeneic tumors in the $C d 4-C r e^{+} L s d 1^{f / f}$ and littermate control mice and found that LSD1 depletion in T cells affected tumor growth variably across tumor models. We observed markedly suppressed growth of MC38 colon carcinoma and TRAMP-C2 prostate adenocarcinoma in contrast to significantly accelerated growth of B16/F10 melanoma, when LSD1 was depleted in T cells (Fig. 1a, b and Supplementary Fig. 2a, b). Interestingly, when a foreign antigen Ovalbumin (OVA) was introduced into B16/F10 cells, this tumor growth acceleration was diminished (Supplementary Fig. 2c), suggesting that tumor immunogenicity could be one of the factors that dictate the anti-tumor or pro-tumor effect of T cell-intrinsic LSD1 depletion. For the following study, we mainly focused on the immunogenic MC38 tumor model, and in some instances also the TRAMP-C2 model, to elucidate the T-cell intrinsic role of LSD1 in antitumor T cell immunity.

To complement the genetic finding in MC38 tumor model, we treated wildtype mice with the LSD1 chemical inhibitor, GSK2879552 30 , daily for two weeks and found that this inhibitor acted similarly as $L s d 1$ genetic ablation, albeit less profoundly, in suppressing tumor growth (Fig. 1c, d). Since our previous study identified an antitumor immune stimulatory effect caused by LSD1 inhibition-induced type I interferon activation in poorly immunogenic tumor models such as B16/F10 and D4m.3 $\mathrm{A}^{31}$, we next sought to determine whether this anti-tumor effect of GSK2879552 came from LSD1 inhibition in tumor or immune cells. Unlike B16/F10 melanoma cells, we found no evidence of interferon pathway activation in MC38 cells treated with LSD1 chemical inhibitors such as GSK-LSD1, which is a tool compound of GSK2879552, and ORY1001 (Fig. 1e). Consistently, MC38 tumors with $L s d 1$ genetic ablation grew similarly as wildtype tumors when implanted in immunocompetent mice (Fig. 1f). Given that specifically targeting LSD1 in MC38 tumor cells had no significant impact on tumor growth in mice with an intact immune system, these findings point to the importance of GSK2879552 on immune cells. To investigate this further, we used $\mathrm{T}$ cell receptor $\alpha$ (TCR $\alpha)$ knockout mice, which are deficient in $\mathrm{CD}^{+}{ }^{+}$and $\mathrm{CD}^{+} \alpha \beta$ T cells, and treated tumor-bearing mice with GSK2879552 as aforementioned. We found GSK2879552 failed to suppress MC38 tumor growth in TCR $\alpha$ KO mice (Fig. 1g, h), suggesting that GSK2879552 acts on LSD1 in T cells to augment the antitumor effect, similar to the impact of $\mathrm{T}$ cellspecific LSD1 depletion, in certain tumors. 
a

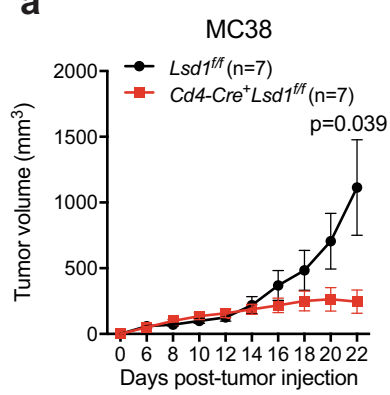

b

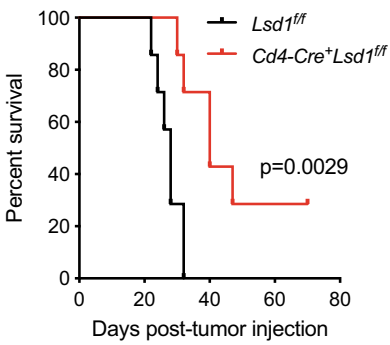

C

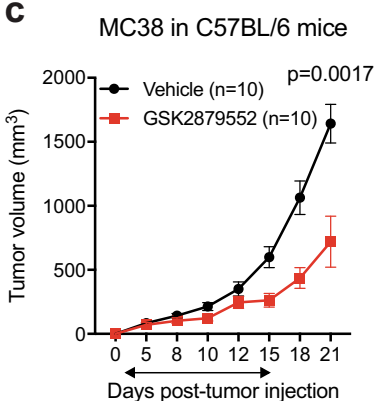

g

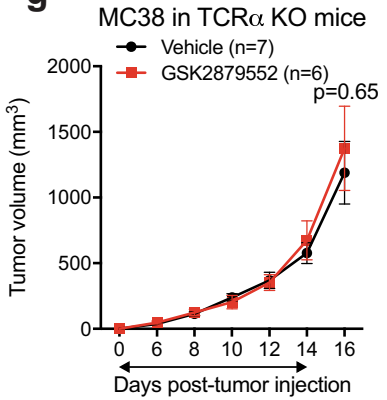

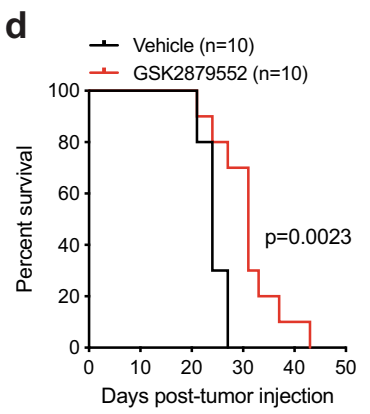

h

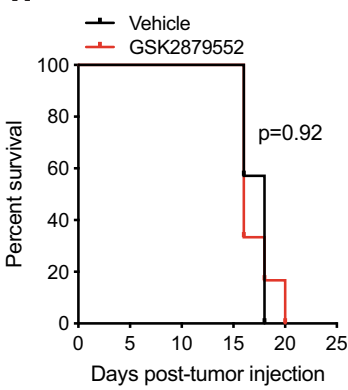

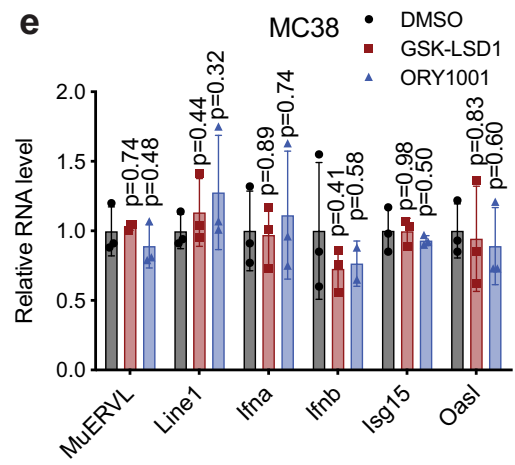

f

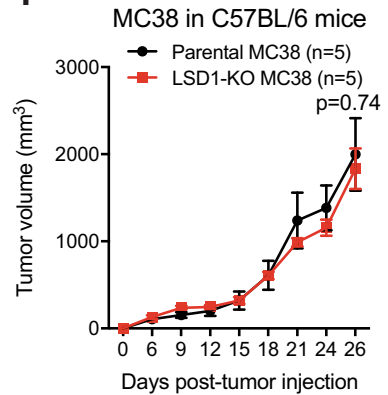

j

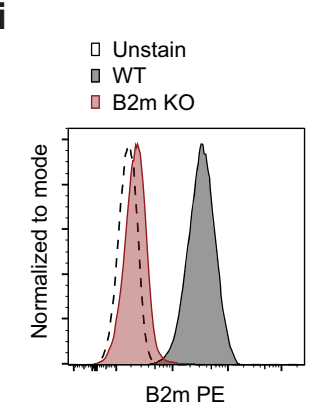

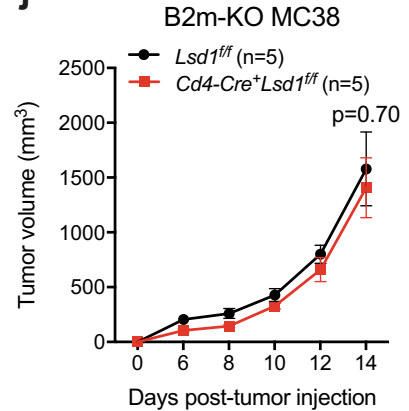

$\mathbf{k}$

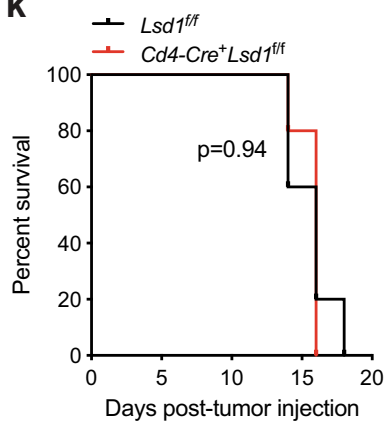

Fig. 1 LSD1 inhibition in T cells enhances antitumor immunity in MC38 tumor model. a, b Tumor growth (a) and survival curves (b) of $L s d f / f$ and Cd4-Cre ${ }^{+}$ Lsd1f/f mice subcutaneously inoculated with MC38 tumor cells ( $n=7$ per group). c, d Tumor growth (c) and survival curves (d) of wildtype mice inoculated with MC38 tumor cells and treated with LSD1 inhibitor GSK2879552 or vehicle control daily for 2 weeks (arrow line). e The real-time qPCR analysis of IFN-related genes in cultured MC38 cells treated with $2 \mu \mathrm{M}$ GSK-LSD1, $0.5 \mu \mathrm{M}$ ORY1001 or vehicle control for 5 days $(n=3)$. f Tumor growth curves of wildtype mice inoculated with wildtype parental or Lsd1 KO MC38 tumor cells. $\mathbf{g}$, $\mathbf{h}$ Tumor growth (g) and survival curves (h) of TCR $\alpha$ KO mice inoculated with wildtype MC38 tumor cells and treated with GSK2879552 or vehicle control daily for 2 weeks (arrow line, vehicle group, $n=7 ;$ GSK2879552 group, $n=6$ ). i Flow cytometry analysis of B2m expression by WT and B2m KO MC38 cells. j, $\mathbf{k}$ Tumor growth (j) and survival curves (k) of $L s d f / f$ and $C d 4-C r e+L s d 7 f / f$ mice inoculated with B2m KO MC38 tumor cells ( $n=5$ per group). Data represent two independent experiments and are presented as mean \pm SEM (standard error of the mean, $\mathbf{a}, \mathbf{c}, \mathbf{f}, \mathbf{g}, \mathbf{j}$ ) or mean $\pm \mathrm{SD}$ (standard deviation, $\mathbf{e}$ ). Sample sizes are as indicated. Statistical significance was determined by two-sided unpaired $\mathrm{t}$ test $(\mathbf{a}, \mathbf{c}, \mathbf{e}-\mathbf{g}, \mathbf{j})$ or log-rank test $(\mathbf{b}, \mathbf{d}, \mathbf{h}, \mathbf{k})$. Source data are provided as a Source data file.

LSD1-deficient $\mathrm{CD8}^{+} \mathrm{T}$ cells demonstrate sustained tumor infiltration, which accounts for the extended tumor growth control. Cytotoxic $\mathrm{CD}^{+} \mathrm{T}$ cells play a central role in antitumor immunity ${ }^{32,33}$. We hypothesized that $\mathrm{CD}^{+} \mathrm{T}$ cells mediated the antitumor effect resulting from $\mathrm{T}$ cell-specific LSD1 depletion in MC38 tumor model. To interrogate this possibility, we deleted the $B 2 m$ gene, which encodes an essential component of the major histocompatibility complex class I (MHC-I) ${ }^{33}$, to abolish tumor antigen recognition by $\mathrm{CD} 8^{+} \mathrm{T}$ cells. We confirmed the loss of $\mathrm{B} 2 \mathrm{~m}$ on MC38 cell surface by flow cytometry (Fig. 1i), and then implanted $\mathrm{B} 2 \mathrm{~m}$-deficient tumors into Cd4-Cre ${ }^{+} \mathrm{Lsd1} / \mathrm{f} / \mathrm{f}$ mice or littermate controls to monitor tumor growth. Unlike wildtype tumors (Fig. 1a, b), B2m-deficient MC38 tumors showed indistinguishable growth kinetics in these two groups of mice (Fig. 1j, k), demonstrating that tumor cell recognition by $\mathrm{CD}^{+} \mathrm{T}$ cells is essential for the antitumor effect caused by $\mathrm{T}$ cell-specific LSD1 depletion. Thus, these results suggest that inhibition of LSD1 in $\mathrm{CD}^{+} \mathrm{T}$ cells potentiates their antitumor ability against certain tumors.

We next investigated how $\mathrm{CD}^{+} \mathrm{T}$ cell immunity in response to tumor growth was regulated by LSD1. The analysis of MC38 tumor growth kinetics suggested that tumor growth can be divided into two stages, an earlier slow growth stage (before day 14) and a later accelerated growth stage (post day 14) (Fig. 1a), which was similarly observed in the TRAMP-C2 tumor model (Supplementary Fig. 2a). Notably, the tumor growth suppression in the $C d 4-C r e^{+} L s d 1^{f / f}$ mice began to appear only when growth in wildtype mice had already entered the acceleration stage (Fig. 1a). To elucidate the immunologic basis underlying this phenotype, 
a

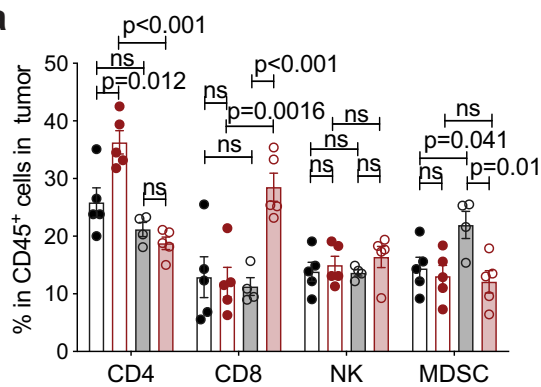

b

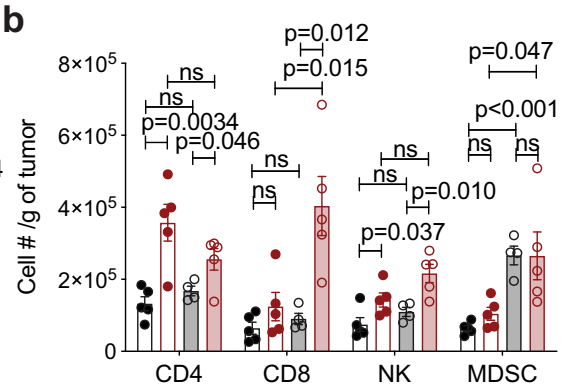

C

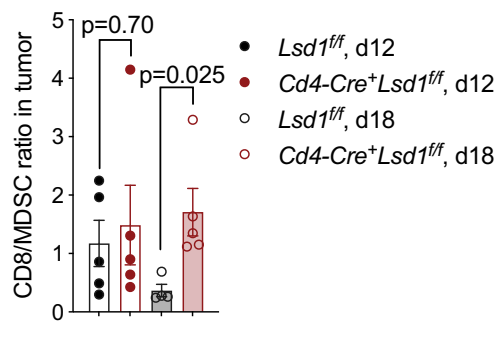

d
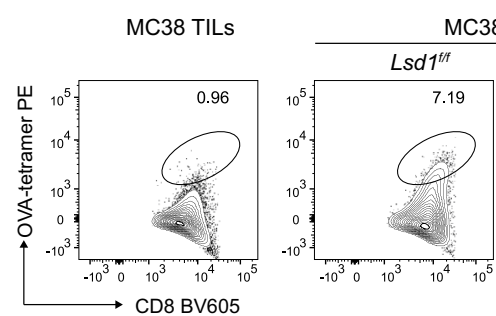

MC38-OVA TILS

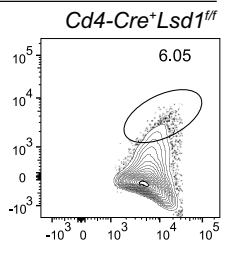

e

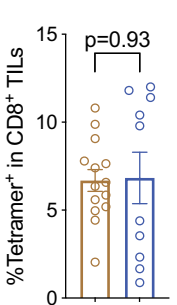

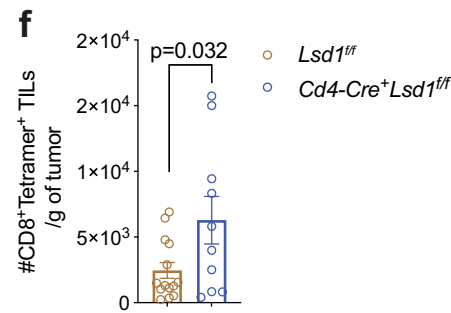

g

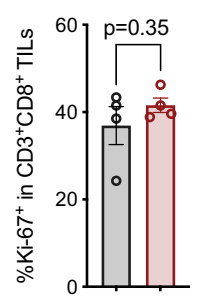

h

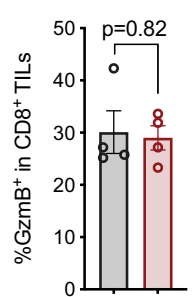

i

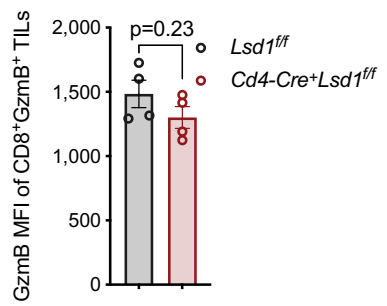

j

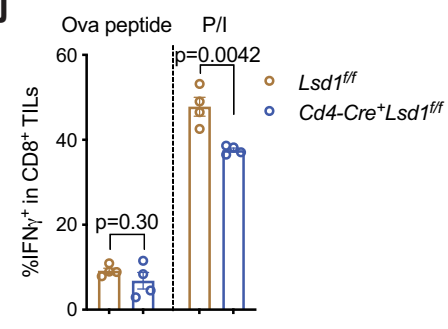

Fig. 2 LSD1 depletion results in sustained tumor infiltration of $\mathbf{C D 8}{ }^{+} \mathbf{T}$ cells. a, b Frequencies of tumor-infiltrating $C D 3^{+} \mathrm{CD} 4^{+} \mathrm{T}$ cells, $\mathrm{CD} 3^{+} \mathrm{CD} 8^{+}$ $\mathrm{T}$ cells, CD3-CD49b+ natural killer (NK) cells and CD11b+Gr1+ myeloid-derived suppressor cells (MDSCs) among gated CD45 ${ }^{+}$leukocytes (a) and cell

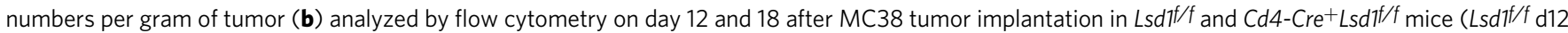

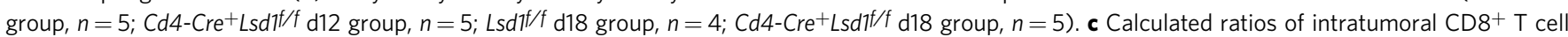
number over MDSCs $\left(n=5,5,4\right.$, or 5, respectively). d-f Representative flow plots (d), percentages (e) and cell numbers (f) of CD8 ${ }^{+}$Tetramer ${ }^{+}$TILs in $^{-}$ implanted MC38-OVA tumors analyzed by flow cytometry on day 18 (Lsd7f/f group, $n=14$; Cd4-Cre+Lsd7f/f group, $n=10$ ). g-i Frequencies of Ki-67expressing CD8 ${ }^{+}$TILs $(\mathbf{g})$, frequencies $(\mathbf{h})$ and mean fluorescence intensity (MFI, i) of GzmB-expressing CD8 ${ }^{+}$TILs analyzed by flow cytometry on day 18 after MC38 tumor implantation ( $n=4$ per group). $\mathbf{j}$ IFN- $\gamma$ expression by MC38-OVA tumor-infiltrating CD8 ${ }^{+}$TILs analyzed by flow cytometry after stimulation with $\mathrm{OVA}_{257-264}$ peptides or PMA/lonomycin (P/I) in the presence of GolgiPlug ( $n=4$ per group). Data represent two independent experiments $(\mathbf{a}-\mathbf{c}, \mathbf{g}-\mathbf{j})$ or are pooled from three independent experiments $(\mathbf{e}, \mathbf{f})$ and are presented as mean $\pm \mathrm{SEM}(\mathbf{a}-\mathbf{j})$. Each dot on the graphs represents an individual mouse. Sample sizes are as indicated. Statistical significance was determined by two-sided unpaired $t$ test; ns, not significant (a-j). Source data are provided as a Source data file.

we analyzed tumor-infiltrating leukocytes (TILs) from MC38 tumor-bearing $\mathrm{Cd} 4-\mathrm{Cre}{ }^{+} \mathrm{Ls} d \mathrm{I}^{\mathrm{f} f f}$ mice and littermate controls at two time points (day 12 and day 18), corresponding to the two stages, which allowed for intra-group longitudinal comparisons as well as inter-group comparisons. In the control Lsd flf mice, antitumor immune populations including $\mathrm{CD}^{+} \mathrm{T}$ cells and natural killer (NK) cells did not show noticeable changes in frequencies or cell numbers when tumors grew from day 12 to day 18 (Fig. 2a, b). Instead, the infiltration of myeloid-derived suppressor cells (MDSCs) was significantly elevated (Fig. 2a, b), in association with the accelerated tumor growth. When LSD1 was depleted in T cells, the infiltration of CD8 ${ }^{+}$TILs remained largely unaltered at the earlier growth stage (day 12), but it was drastically increased at the later time point (day 18) (Fig. 2a, b), making the ratio of $\mathrm{CD}^{+}$TILs cell number over MDSCs significantly higher in the Cd4-Cre $\mathrm{Lsd}^{\mathrm{f} / \mathrm{f}}$ mice than that in the littermate controls (Fig. 2c).

Using Ovalbumin-expressing MC38 tumors (MC38-OVA) and tetramer staining, we found that the percentage of OVA-specific
$\mathrm{T}$ cells among total $\mathrm{CD}^{+}$TILs was comparable between these two groups of mice (Fig. 2d, e). Thus, tumor antigen-specific $\mathrm{T}$ cells were correspondingly expanded along with the increased total $\mathrm{CD}^{+}$TILs in response to LSD1 depletion on day 18 (Fig. 2f). In addition, although the total number of $\mathrm{CD} 8^{+} \mathrm{T}$ cells in tumor-draining lymph nodes (TdLNs) was reduced by LSD1 depletion (Supplementary Fig. 3a-d), OVA-specific CD ${ }^{+} \mathrm{T}$ cells in TdLNs were numerically comparable between the two groups of mice carrying MC38-OVA tumors (Supplementary Fig. 3e, f), suggesting $\mathrm{CD} 8^{+} \mathrm{T}$ cell priming in response to the tumor growth is mostly unaffected by LSD1 depletion. As aforementioned, LSD1-competent and -deficient $\mathrm{CD} 8^{+} \mathrm{T}$ cell infiltration was comparable on day 12 (Fig. 2a, b), suggesting that $\mathrm{CD}^{+} \mathrm{T}$ cell recruitment to the tumor sites is also unlikely affected by LSD1 depletion, at least at the earlier stage. Thus, we speculated that LSD1 depletion may enhance the local expansion of CD8 ${ }^{+}$TILs after their recruitment into the tumor microenvironment (TME), and this speculation is consistent with the recent studies demonstrating the importance of intratumoral $\mathrm{CD}^{+} \mathrm{T}$ cell 
a

MC38 implantation

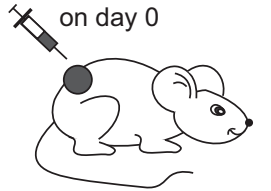

b

Gated on PBMCs:
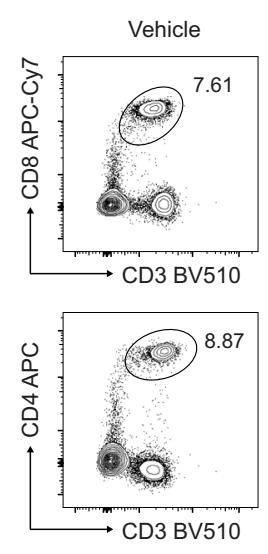
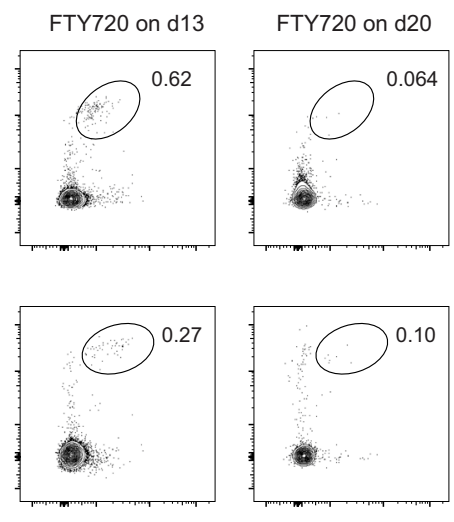

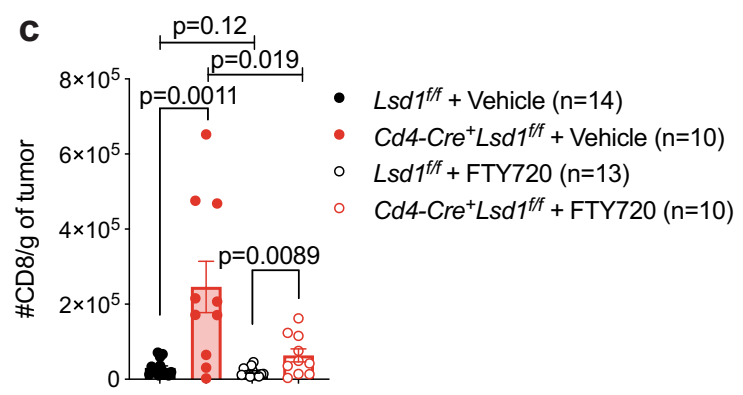

d

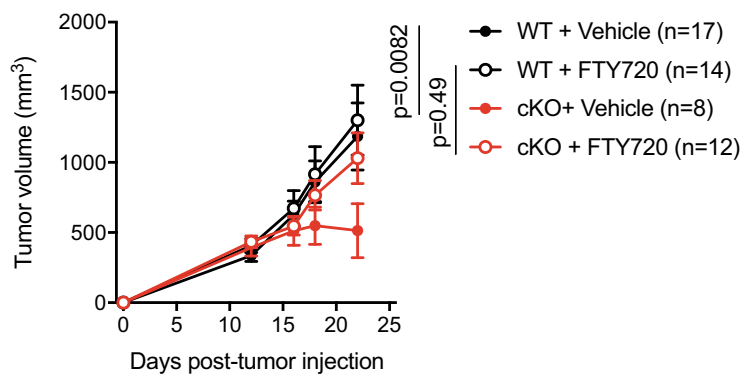

e

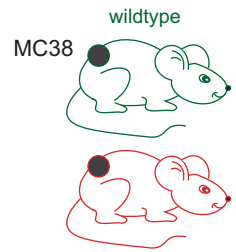

$\mathrm{Cd} 4-\mathrm{Cre}^{+} \mathrm{Lsd} 1^{\mathrm{t} / \mathrm{t}}$
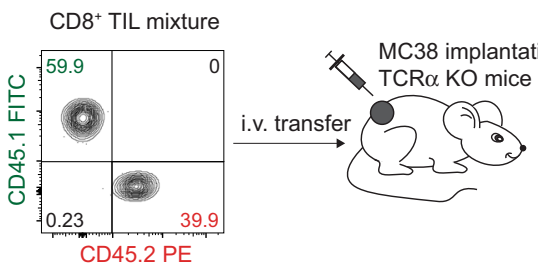

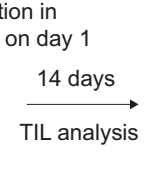

IL analysis

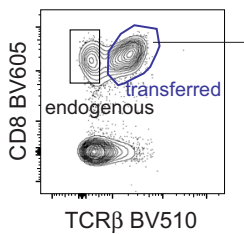

TCR $\beta$ BV510

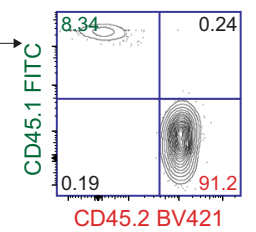

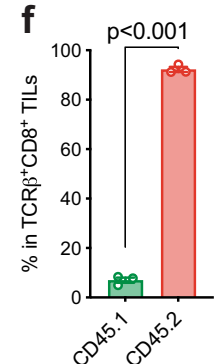

Fig. 3 LSD1 loss promotes intratumoral CD8 ${ }^{+} \mathbf{T}$ cell persistence. a Experimental design of administering FTY720 to block T cell egress. b Representative flow plots showing frequencies of $\mathrm{CD}^{+}$and $\mathrm{CD} 8^{+} \mathrm{T}$ cells in PBMCs of mice receiving FTY720 or vehicle. c Cell numbers of CD8 ${ }^{+}$TILs on day 22 . $\mathbf{d}$ Tumor growth curves of WT and Cd4-Cre ${ }^{+}$Lsd $f^{f / f}$ (cKO) mice implanted with MC38 tumors and treated with FTY720 or vehicle. e Experimental design of CD8 ${ }^{+}$TILs co-transfer and assessment of intratumoral persistence. $\mathbf{f}$ Frequencies of CD45.1+ wildtype and CD45.2+ LSD1-deficient cells among transferred TCR $\beta^{+} \mathrm{CD} 8+$ TILs $(n=3$ per group). Data are pooled from two experiments and presented as mean \pm SEM (c, d, f). Sample sizes are as indicated. Statistical significance was determined by two-sided unpaired $t$ test $(\mathbf{c}, \mathbf{f})$ or two-way ANOVA (d). Source data are provided as a Source data file.

expansion in tumor control in response to a variety of stimuli ${ }^{7,8,34}$. The effector function of MC38 tumor-infiltrating $\mathrm{CD}^{+} \mathrm{T}$ cells, however, appeared largely unaffected as we detected no overt changes in Ki-67 and granzyme B (GzmB) expression and only a modest reduction of IFN- $\gamma$ expression upon LSD1 depletion (Fig. 2g-j). Taken together, our data suggest that loss of LSD1 in T cells improves the antitumor effect through elevating intratumoral $\mathrm{CD}^{+} \mathrm{T}$ cell accumulation, potentially mediated by their local expansion.

LSD1 loss promotes intratumoral $\mathrm{CD8}^{+} \mathrm{T}$ cell expansion. To examine the local expansion of $\mathrm{CD} 8^{+} \mathrm{T}$ cells in the TME and its regulation by LSD1, we implanted MC38 tumors in $\mathrm{Cd} 4-\mathrm{Cr} \mathrm{C}^{+}$ Lsd $1 / f$ mice and littermate controls for 12 days to allow initial $\mathrm{T}$ cell infiltration in comparable numbers and then treated those mice with FTY720, a S1P receptor agonist that can block additional $\mathrm{T}$ cell recruitment from lymph nodes to tumor sites (Fig. 3a). FTY720 administration effectively blocked $\mathrm{T}$ cell egress (Fig. $3 \mathrm{~b}$ ), and brought down the number of $\mathrm{CD} 8^{+}$TILs compared with vehicle control in Cd4-Cre ${ }^{+} L s d 1^{f / f}$ mice (Fig. 3c). In line with this, the inhibition of continued $\mathrm{T}$ cell recruitment attenuated the antitumor effect of LSD1 depletion (Fig. 3d). Nevertheless, LSD1-deficient $\mathrm{CD}^{+}$TILs still showed a significantly higher cell number than wildtype CD8 ${ }^{+}$TILs in response to FTY720 treatment (Fig. 3c). These results suggest that, while continued $\mathrm{CD}^{+} \mathrm{T}$ cell recruitment is a critical basis for antitumor immunity, LSD1 depletion acts on the recruited $\mathrm{CD} 8^{+}$ $\mathrm{T}$ cells to enhance their intratumoral expansion. Whether LSD1 depletion also promoted $\mathrm{CD}^{+} \mathrm{T}$ cell recruitment particularly at the later stage of tumor growth remained to be further addressed.

To further evaluate the expansion ability of tumor-resident CD8 ${ }^{+}$ $\mathrm{T}$ cells with or without LSD1, we isolated CD8 ${ }^{+}$TILs from MC38 tumor-bearing $\mathrm{Cd} 4-\mathrm{Cre} e^{+} \mathrm{Ls} d \mathrm{I}^{\mathrm{f} f f}\left(\mathrm{CD} 45.2^{+}\right)$and congenic wildtype $\left(\mathrm{CD} 45.1^{+}\right)$mice and co-transferred those cells into TCRa KO recipient mice receiving MC38 tumors, followed by TIL analysis 14 days later (Fig. 3e). Among the transferred $\mathrm{CD} 8^{+}$TILs $\left(\mathrm{TCR} \beta^{+}\right)$, LSD1-deficient cells drastically exceeded wildtype cells in numbers, despite the fact that similar numbers of cells were co-transferred (Fig. $3 \mathrm{f}$ ), again supporting that the intratumoral $\mathrm{CD}^{+} \mathrm{T}$ cells lacking LSD1 acquired better expansion capability.

LSD1 depletion preserves the TCF ${ }^{+}$PD- $1^{\text {int }}$ progenitor subset of exhausted $\mathrm{CDB}^{+} \mathrm{T}$ cells in the TME. What is the biological basis that underlies the sustained expansion of LSD1-deficient $\mathrm{CD}^{+}$TILs? Recent studies have documented that among 
exhausted $\mathrm{CD}^{+} \mathrm{T}$ cells, the progenitor $\mathrm{CD}^{+} \mathrm{T}$ cells coexpressing PD-1 at an intermediate level (PD- $1^{\text {int }}$ ) and TCF1 (encoded by $T c f 7$ gene) essentially sustain intratumoral $\mathrm{T}$ cell expansion through self-renewing while continuously giving rise to numerically larger TCF1-negative, more differentiated cells ${ }^{6,8}$. In line with those reports, we showed that isolated CD8 ${ }^{+}$TILs with the PD- ${ }^{\text {int }}$ phenotype, when adoptively transferred into MC38 tumor-bearing mice, retained a limited but detectable number of TCF $1^{+} \mathrm{PD}-1^{\text {int }}$ cells in the TME, while converting into numerically more TCF $1^{-} \mathrm{PD}-1^{\text {hi }}$ cells than did transferred PD-1 hi cells (Supplementary Fig. $4 \mathrm{a}-\mathrm{g}$ ). We thus set out to examine whether the progenitor exhausted CD ${ }^{+} \mathrm{T}$ cells were possibly regulated by LSD1. PD-1 expression, which is induced and sustained by chronic antigen stimulation ${ }^{2}$, was activated in both wildtype and LSD1-deficient $\mathrm{CD}^{+}$TILs (Fig. 4a). Although similar frequencies of PD-1 ${ }^{+}$cells were detected (Fig. $4 \mathrm{a}$ ), the cell surface $\mathrm{PD}-1$ protein in LSD1-deficient CD8 ${ }^{+}$TILs was expressed at a significantly lower level compared with that in wildtype $\mathrm{CD} 8^{+}$ TILs of MC38 tumors (Fig. 4b). Among wildtype CD8 ${ }^{+}$TILs, TCF $1{ }^{+} \mathrm{PD}-1^{\text {int }}$ cells represented a subset much smaller than PD1 ${ }^{\text {hi }}$ cells, which by and large lost TCF1 expression (TCF1-PD-

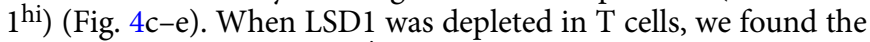
percentage of $\mathrm{TCF} 1^{+} \mathrm{PD}-1^{\text {int }}$ cells significantly increased while the percentage of TCF $1^{-} \mathrm{PD}-1^{\text {hi }}$ cells correspondingly decreased compared with their wildtype counterparts on both day 12 and 18 (Fig. 4d). Importantly, a larger cellular expansion of TCF1+PD1 int TILs from day 12 to day 18 was observed when LSD1 was abrogated, which was associated with a greater expansion of the TCF1-PD-1 ${ }^{\text {hi }}$ TILs, resulting in increased absolute cell numbers for both the $\mathrm{TCF} 1^{+} \mathrm{PD}-1^{\mathrm{int}}$ and $\mathrm{TCF} 1^{-} \mathrm{PD}-1^{\text {hi }}$ subsets in the absence of LSD1 (Fig. 4e). In contrast, the TCF $1^{+} \mathrm{PD}-1^{\text {hi }}$ subset was not elevated by LSD1 depletion (Fig. 4d, e), thus unlikely responsible for the increased expansion of LSD1-deficient CD8 ${ }^{+}$ TILs, even though this subset might also contain the progenitor exhausted CD8 ${ }^{+} \mathrm{T}$ cells. Using MC38-OVA and tetramer staining, we further showed that tumor antigen-specific CD8 ${ }^{+}$TILs consistently displayed an elevated $\mathrm{TCF} 1^{+} \mathrm{PD}-1^{\text {int }}$ subset in response to LSD1 loss (Fig. $4 \mathrm{f}$ ), while in TdLNs, TCF $1^{+} \mathrm{PD}-1^{\text {int }}$ subset remained numerically unaltered (Supplementary Fig. 5a-c). When $\mathrm{T}$ cell recruitment was blocked by FTY720 treatment starting on day 12 post tumor implantation, the TCF $1{ }^{+}$PD- $1^{\text {int }}$ subset of LSD1-deficient CD8 ${ }^{+}$TILs appeared to be preferentially decreased to a level comparable to that of the WT counterpart in the next 10 days, while expectedly giving rise to numerically more TCF1-PD-1 ${ }^{\text {hi }}$ cells than the WT counterpart (Supplementary Fig. $6 \mathrm{a}-\mathrm{c}$ ). Collectively, the TCF $1+\mathrm{PD}-1^{\text {int }}$ subset of exhausted $\mathrm{CD} 8^{+}$TILs seem to gain a stronger, but nonetheless limited, expansion ability upon LSD1 depletion, which, therefore, also underscores the need and importance of constant replenishment from TdLNs.

In addition to the MC38 tumor model, we found that LSD1 depletion also led to the expansion of $\mathrm{TCF} 1^{+} \mathrm{PD}-1^{\text {int }} \mathrm{CD} 8^{+}$TILs in pool size in the TRAMP-C2 tumor model, which was associated with the increased $\mathrm{CD}^{+} \mathrm{T}$ cell infiltration and the decreased PD-1 expression level by CD8 ${ }^{+}$TILs (Supplementary Fig. 7a-g). In B16/F10 tumor model, a substantial percentage $(>30 \%)$ of $\mathrm{CD}^{+}$TILs showed the progenitor phenotype (PD-1 $\left.{ }^{\text {int }}\right)$, which was further elevated close to $60 \%$ by LSD 1 depletion (Supplementary Fig. 8a-c). Thus, a majority of LSD1deficient $\mathrm{CD}^{+}$TILs retained the progenitor phenotype, which possibly compromises the timely conversion to more differentiated PD- 1 hi cells that are responsible for generating cytotoxicity and immediate tumor control, contributing to an accelerated B16/ F10 tumor growth. Indeed, the GzmB expression by CD8 ${ }^{+}$TILs was profoundly decreased by LSD1 depletion (Supplementary Fig. 8d, e). Introducing an OVA antigen to B16 tumors
(B16-OVA) appeared to mitigate the defect in GzmB expression likely through promoting the conversion of PD-1 $1^{\text {int }}$ cells to PD$1^{\text {hi }}$ cells (Supplementary Fig. 8f-j). Therefore, LSD1 depletion in $\mathrm{T}$ cells consistently elevates the progenitor subset of exhausted $\mathrm{CD}^{+} \mathrm{T}$ cell in the TME of a variety of tumor models, in spite of its differential impacts on tumor growth.

To ensure that the phenotype of $\mathrm{CD}^{+}$TILs was modulated by intrinsic LSD1 loss irrespective of $\mathrm{CD} 4^{+} \mathrm{T}$ cells, we used splenic $\mathrm{CD}^{+} \mathrm{T}$ cells from $\mathrm{Cd} 4-\mathrm{Cr} e^{+}{ }_{\mathrm{Ls}}{ } 1^{f / f}$ mice and littermate controls to reconstitute TCRa KO mice respectively, which were later implanted with MC38 tumors (Supplementary Fig. 9a). The analysis of $\mathrm{CD}^{+}$TILs on day 20 recapitulated the phenotype that LSD1-deficient $\mathrm{CD}^{+} \mathrm{T}$ cells were more expanded in the TME and displayed an elevated $\mathrm{TCF} 1^{+} \mathrm{PD}-1^{\text {int }}$ subset (Supplementary Fig. 9b-d). Together, the above results demonstrate that LSD1 loss in $\mathrm{CD}^{+} \mathrm{T}$ cells intrinsically helps preserve the $\mathrm{TCF} 1^{+} \mathrm{PD}-$ $1^{\text {int }}$ progenitor exhausted cells.

We next examined the impact of LSD1 loss on different subsets of $\mathrm{CD}^{+}$TILs. Slamf6, recently reported as a marker of progenitor exhausted $\mathrm{T}$ cells ${ }^{6}$, was preferentially expressed by TCF $1+$ PD -1 int and TCF $1{ }^{+} \mathrm{PD}-1$ hi cells, and its expression was upregulated by LSD1 depletion across all subsets (Fig. 4g). A terminal exhaustion marker, Tim3, was significantly less expressed in $\mathrm{TCF} 1^{+} \mathrm{PD}-1^{\text {int }}$ cells than $\mathrm{TCF} 1^{+} \mathrm{PD}-1$ hi cells (Fig. 4h), in line with the view that $\mathrm{TCF} 1^{+} \mathrm{PD}-1^{\text {int }}$ cells represent the progenitor exhausted subset. Of note, LSD1 loss specifically downregulated Tim 3 expression in the TCF $1^{+} \mathrm{PD}-1^{\text {int }}$ subset but not in others (Fig. 4h). Additionally, in line with a recent report ${ }^{35}$, Ly6C expression was detected in a major part of the TCF1 ${ }^{+} \mathrm{PD}$ $1^{\text {int }}$ subset, whereas only a minor part of the $\mathrm{TCF} 1^{+} \mathrm{PD}-1^{\text {hi }}$ as well as TCF1-PD-1 $1^{\text {hi }}$ subsets expressed Ly6C (Fig. 4i, j), indicating that Ly6C could be an additional marker to distinguish the TCF $1^{+} \mathrm{PD}$ $1^{\text {int }}$ progenitor exhausted subset from others. Upon LSD1 loss, Ly6C expression was elevated in the $\mathrm{TCF} 1^{+} \mathrm{PD}-1^{\text {int }}$ subset and sustained even in the TCF1-PD-1 ${ }^{\text {hi }}$ subset (Fig. 4i, j). The TCF $1{ }^{+} \mathrm{PD}-1^{\text {int }}$ progenitor exhausted subset expressed much less GzmB than did the more differentiated TCF1-PD-1 hi subset in which GzmB expression was not interrupted by LSD1 loss (Fig. 4k). The expression of a proliferation marker Ki-67 remained largely unaltered by LSD1 depletion across all subsets (Fig. 4l). The unaltered proliferation rate of the TCF $1^{+} \mathrm{PD}-1^{\text {int }}$ subset in response to LSD1 depletion suggests a potential survival advantage underlying the intratumoral persistence of these cells. Compared with $\mathrm{CD} 8^{+} \mathrm{PD}-1^{-}$TILs, antigen-experienced PD- $1^{\text {int }}$ and PD-1 ${ }^{\text {hi }}$ cells showed higher levels of apoptosis (Fig. $4 \mathrm{~m}, \mathrm{n}$ ). Indeed, LSD1-deficient PD-1 ${ }^{\text {int }}$ cells showed significantly less Annexin V staining and lower Caspase 3/7 activation than their wildtype counterparts (Fig. $4 \mathrm{~m}, \mathrm{n}$ ). Importantly, such resistance was not observed in the PD- 1 hi cells that mostly have lost TCF1 expression (Fig. 4m, n), indicating a unique mechanism of action of LSD1 that is restricted to the TCF1-expressing CD8 ${ }^{+}$TILs. Taken together, these results suggest that LSD1 depletion selectively improves the survival of the progenitor subset of exhausted $\mathrm{CD}^{+}$TILs contributing to the generation of an increasing number of more differentiated cells with better cytotoxicity.

The LSD1/CoREST complex physically interacts with TCF1 and antagonizes its transcriptional activity. TCF1 is a central transcription factor in maintaining the progenitor phenotype of the $\mathrm{CD}^{+}{ }^{+} \mathrm{TCF} 1^{+} \mathrm{PD}-1^{\text {int }} \mathrm{T}$ cells in tumors and chronic viral infections ${ }^{6-8,11,13,36}$. The observation that LSD1 acts selectively in the $\mathrm{TCF} 1^{+} \mathrm{PD}-1^{\text {int }}$ cells prompted us to investigate possible interplays between LSD1 and TCF1. We first asked whether LSD1 regulated TCF1 expression and found that, in the 

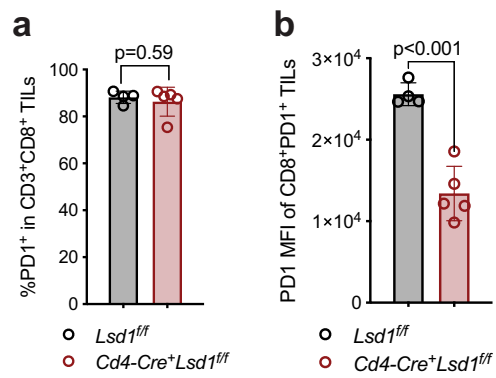

C

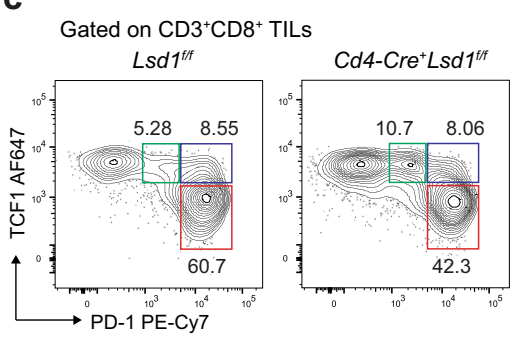

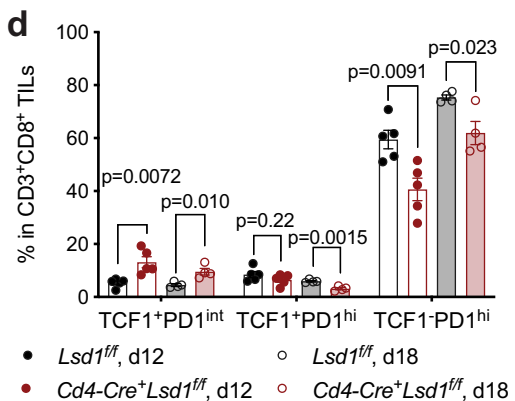
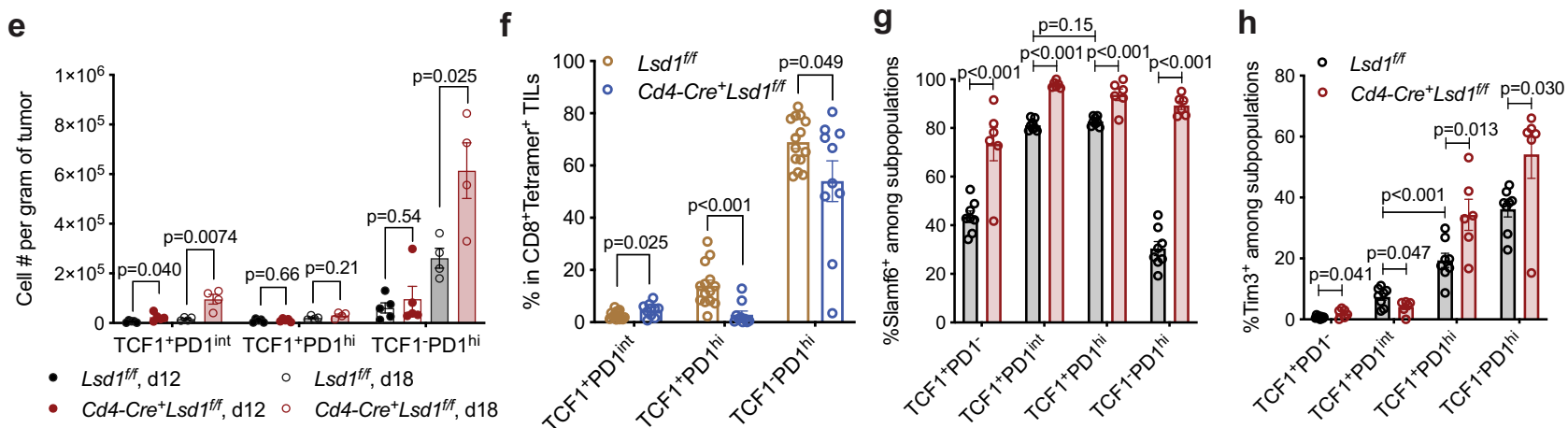

i
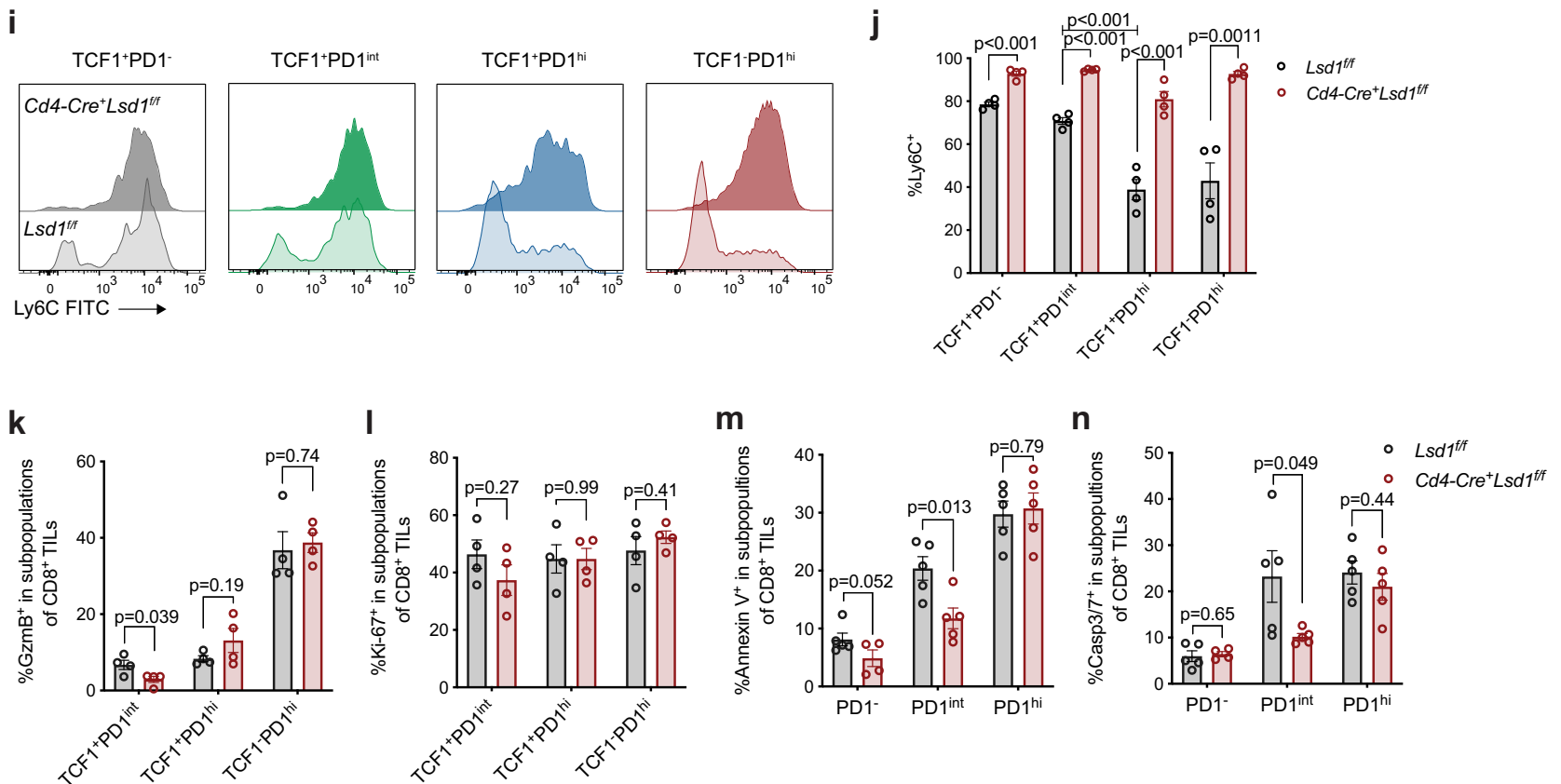

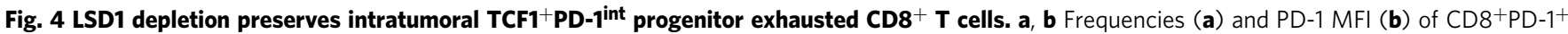
TILs on day 18 after MC38 tumor implantation ( $L s d f^{f / f}$ group, $n=4 ; C d 4-C r e^{+} L s d 1^{f / f}$ group, $n=5$ ). c-e Representative flow plots (c), percentages (d) and cell numbers (e) of TCF1+PD-1 $1^{\text {int }}$, TCF1+PD- $1^{\text {hi }}$ and TCF1-PD- $1^{\text {hi }}$ cells in CD8 ${ }^{+}$TILs analyzed by flow cytometry on day 12 and 18 after MC38 tumor implantation (Lsd1f/f d12 group, $n=5 ; C d 4-C r e^{+} L s d 1 f / f$ d12 group, $n=5$; Lsd $7 / f$ d18 group, $n=4 ; C d 4-C r e+L s d 1 / f$ d18 group, $n=4$ ). f Percentages of TCF1+PD-1int, TCF1+PD-1hi and TCF1-PD-1hi cells in CD8 ${ }^{+}$Tetramer+ TILs on day 18 after MC38-OVA tumor implantation (Lsd7f/f group, $n=14 ;$ Cd4-Cre $^{+}$ Lsd $7 / f$ group, $n=10) . \mathbf{g}$, h, Slamf6 $(\mathbf{g})$ and Tim3 $(\mathbf{h})$ expression by different subpopulations of CD8 ${ }^{+}$TILs analyzed by flow cytometry (Lsd7f/f group, $n=8$; Cd4-Cre+ Lsd f/f group, $n=6$ ). $\mathbf{i}$, j, Representative flow plots of Ly6C expression (i) and percentages of Ly6C $C^{+}$cells (j) in different subpopulations of CD8 ${ }^{+}$ TILs ( $n=4$ per group). $\mathbf{k}, \mathbf{I}$ GzmB (k) and Ki-67 (I) expression by different subpopulations of CD8 ${ }^{+}$TILs analyzed by flow cytometry on day 18 ( $n=4$ per group). $\mathbf{m}, \mathbf{n}$ Cell apoptosis analysis by Annexin $\bigvee$ staining $(\mathbf{m})$ and active Casp3/7 staining $(\mathbf{n})$ of CD8 ${ }^{+}$TILs isolated from MC38 tumors on day 14 ( $n=5$ per group). Data represent two independent experiments (a-e, $\mathbf{g}-\mathbf{n}$ ) or are pooled from three independent experiments (f) and are presented as mean \pm SEM (a-n). Statistical significance was determined by two-sided unpaired t test (a-n). Source data are provided as a Source data file.

CD8 ${ }^{+} \mathrm{TCF} 1^{+} \mathrm{PD}-1^{\text {int }}$ TILs, the protein level of TCF1 was not affected by LSD1 loss (Fig. 5a). We next asked whether LSD1 may impact TCF1 transcriptional activity through protein-protein interactions, as LSD1 is known to interact with a number of
DNA-binding transcription factors ${ }^{37}$. Indeed, in HEK293T cells, antibodies against tagged LSD1 co-immunoprecipitated TCF1 protein, as well as CoREST and HDAC1, which are known components of the LSD1/CoREST complex (Fig. 5b) ${ }^{38}$. 
a

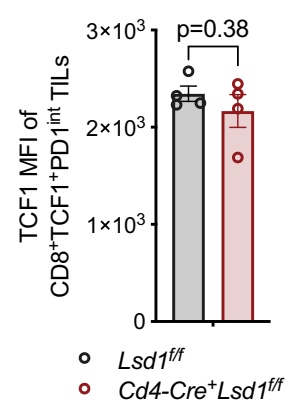

b

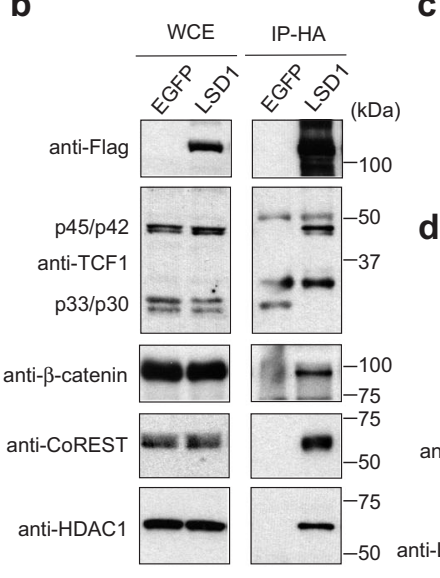

C

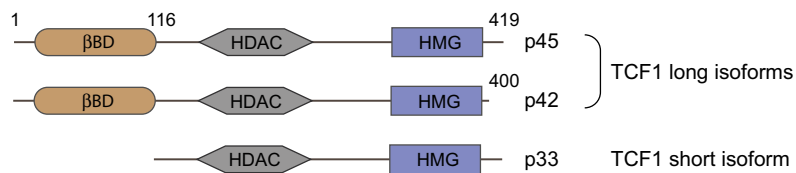

d
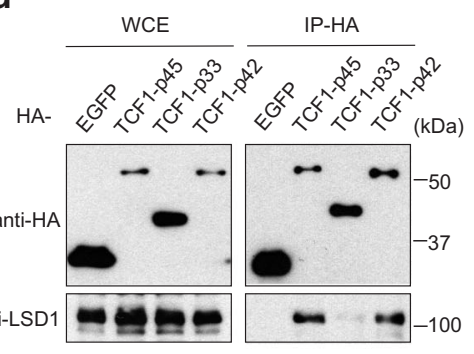

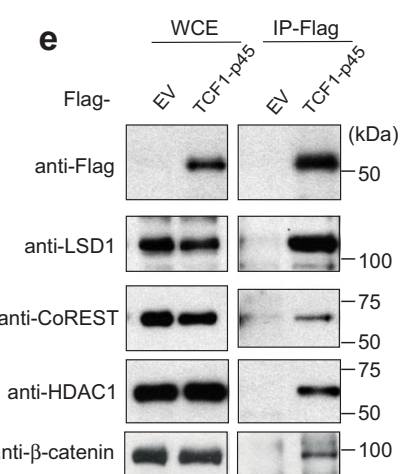

f

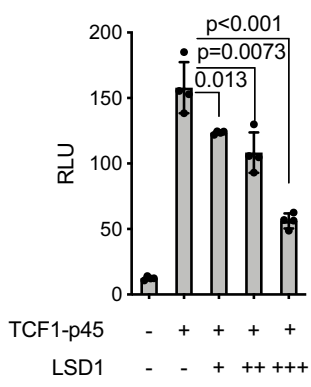

g

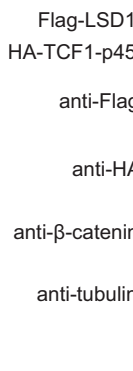

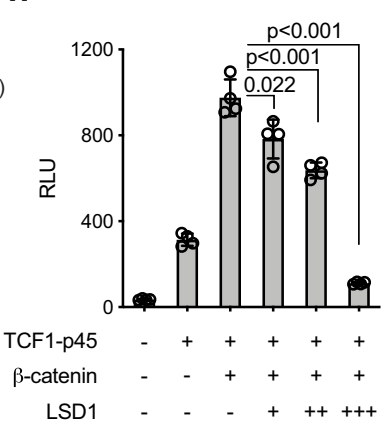

i

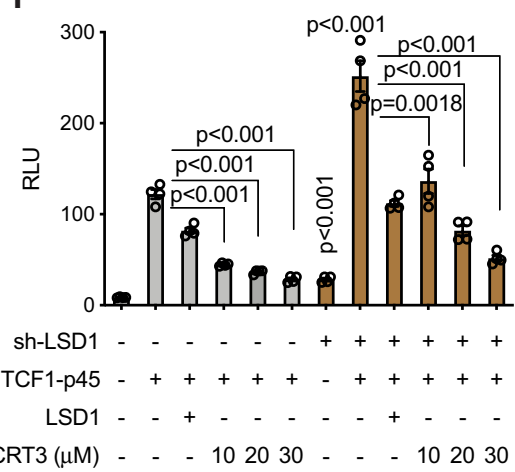

j

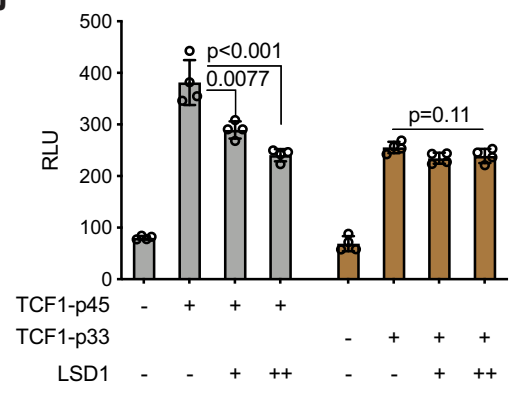

k

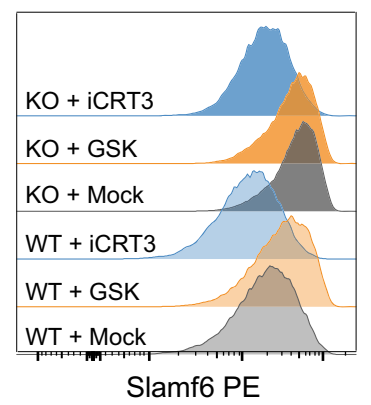

I

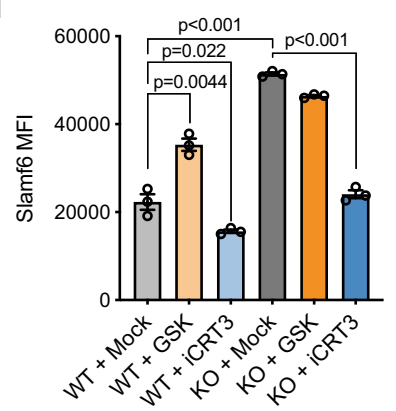

Fig. 5 LSD1/CoREST complex physically interacts with TCF1 and antagonizes its transcriptional activity. a MFI of TCF1 in CD8 ${ }^{+}$TCF1+PD- $1^{\text {int }}$ TILs analyzed by flow cytometry ( $n=4$ per group). $\mathbf{b}$ The physical interaction between LSD1 and TCF1 isoforms as well as other proteins was examined by coimmunoprecipitation (co-IP) assays using whole-cell extract (WCE) of HEK293T cells transfected with FLAG-HA-tagged LSD1. c Schematic representation of different isoforms of mouse TCF1. d Co-IP assays precipitating HA-tagged TCF1 isoforms and detecting LSD1 in eluted IP fractions. e Co-IP assays precipitating Flag-tagged TCF1 (referring to p45 unless otherwise specified) and detecting components of the LSD1/CoREST complex in eluted IP fractions. $\mathbf{f}-\mathbf{j}$ TCF/LEF reporter assay measuring relative luciferase units (RLU) in response to transfection with plasmids expressing indicated proteins $(\mathbf{f}-\mathbf{j}, n=4$ per condition) or shRNA targeting LSD1 (i) and the treatment with iCRT3 (i) ( $n=4$ per condition). Protein expression for plasmid transfection in $\mathbf{f}$ is shown by immunoblotting (g). k, I Representative flow plots (k) and Slamf6 MFI (I) of splenic CD8 ${ }^{+}$T cells activated in vitro for 3 days with anti-CD3/anti-CD28 in the presence of vehicle, GSK2879552 or iCRT3 ( $n=3$ per treatment). Data represent two $(\mathbf{a}, \mathbf{b}, \mathbf{d}-\mathbf{j})$ or three (k, $\mathbf{l})$ independent experiments and are presented as mean \pm SEM (a) or mean \pm SD $(\mathbf{f}, \mathbf{h}-\mathbf{j}, \mathbf{I})$. Statistical significance was determined by two-sided unpaired $t$ test $(\mathbf{a}, \mathbf{f}, \mathbf{h}-\mathbf{j}, \mathbf{I})$. Source data are provided as a Source data file.

Interestingly, only the long isoforms of TCF1-p45/p42 with the $\beta$ catenin binding domain ( $\beta B D$ ) was detected in the LSD1 immunoprecipitates, though both long (p45/p42) and short (p33 and p30) isoforms of TCF1 were expressed in these cells (Fig. 5b, c). In addition, $\beta$-catenin, which is a binding partner of the TCF1 long isoform important for TCF1-mediated transcriptional activation $^{39}$, was also present in the LSD1 immunoprecipitates (Fig. 5b). To further confirm the selective interaction of LSD1 with the TCF1 long isoform, we expressed the epitope-tagged, TCF1 long isoform (p45 and p42) and short isoform (p33) for reciprocal co-immunoprecipitation (co-IP), respectively. We found that LSD1 protein was only present in the immunoprecipitates of the 
long isoform but not the short isoform of TCF1 (Fig. 5d), suggesting that the physical interaction of TCF1 with LSD1 involves the $\beta \mathrm{BD}$ domain present only in the TCF1 long isoform. However, whether LSD1 or other components of the LSD1/CoREST complex directly bind to the $\beta \mathrm{BD}$ domain of TCF1 long isoform or indirectly through its binding to $\beta$-catenin or other TCF1interacting proteins remains to be further investigated. As expected, several other components of the LSD1/CoREST complex were also pulled down by the TCF1 long isoform in the co-IP assays (Fig. 5e). Thus, these results suggest that the LSD1/CoREST complex physically interacts with the long isoform of TCF1.

LSD1 represses gene expression by catalyzing the removal of the methyl groups from histone $\mathrm{H} 3 \mathrm{~K} 4 \mathrm{mel} / 2^{40}$. The fact that LSD1 interacts with TCF1 raised the possibility that LSD1 may compromise TCF1-mediated transcriptional activation. To test this possibility, we used a well-established reporter assay designed to monitor the transcriptional activation mediated by TCF/LEF family members ${ }^{41}$. Indeed, the transcriptional activity of the TCF1 long isoform was suppressed by the co-transfected LSD1 in a dose-dependent manner without altering protein expression of the ectopic TCF1 or endogenous $\beta$-catenin (Fig. 5f, g). Co-transfection of $\beta$-catenin strongly elevated the transcriptional activity of the TCF1 long isoform, which could also be largely attenuated by overexpressing LSD1 (Fig. 5h). Conversely, LSD1 downregulation significantly increased the transcriptional activity mediated by the endogenous TCF/LEF as well as the ectopically expressed TCF1 long isoform (Fig. 5i). $\beta$-catenin is a transcriptional co-activator of TCF1 and the physical interaction of $\beta$-catenin with TCF1 is important for transcriptional regulation, as disruption of their interaction by the inhibitor, iCRT $3^{42}$, severely dampened the transcriptional activation mediated by TCF1/ $\beta$-catenin (Fig. 5i). Our findings suggest that LSD1 compromises TCF1-mediated transcription by physically interacting with TCF1. Consistent with this hypothesis, the transcriptional activity of the TCF1 short isoform, which does not interact with LSD1, was not affected by changes of LSD1 protein levels (Fig. 5j). Taken together, these data suggest that the physical interaction of LSD1 with TCF1 suppresses its transcriptional activity, although detailed molecular mechanisms remain to be elucidated.

To determine the biological significance of this interplay in $\mathrm{T}$ cells, we activated $\mathrm{CD} 8^{+} \mathrm{T}$ cells isolated from mouse spleens by polyclonal stimulation and examined the transcriptional activity of the endogenous TCF1 by measuring the expression of its target gene Slamf $6^{6,11}$. As a control. disrupting TCF1/ $\beta$-catenin complex by iCRT3 compromised Slamf6 induction in activated CD8 ${ }^{+}$ $\mathrm{T}$ cells as expected (Fig. $5 \mathrm{k}, 1)$. In contrast, inhibition of LSD1 by GSK2879552 during $T$ cell activation significantly upregulated Slamf6 expression, which was recapitulated by LSD1 genetic ablation (Fig. 5k, l). Thus, LSD1 antagonizes the transcriptional activity of TCF1 in $\mathrm{CD} 8^{+} \mathrm{T}$ cells.

LSD1 inhibition unleashes TCF1-controlled gene expression involved in intratumoral $\mathrm{T}$ cell persistence. On the basis of the above data, we hypothesized that LSD1 loss augmented the transcriptional program mediated by TCF1 that maintained a progenitor subset of intratumoral $\mathrm{CD}^{+} \mathrm{T}$ cells for extended tumor control. To address this possibility, we isolated MC38 tumor-infiltrating $\mathrm{CD} 8{ }^{+} \mathrm{CD} 44^{+} \mathrm{PD} 1^{+} \mathrm{T}$ cells for transcriptomic analysis by RNA-seq (Supplementary Fig. 10a, b). We found 561 genes upregulated and 351 genes downregulated (fold change $>$ 1.5 and $\mathrm{FDR}<0.01$ ) in LSD1-deficient versus wildtype cells (Fig. 6a and Supplementary Data 1), and the differentially expressed genes were enriched in immune-related pathways (Supplementary Fig. 10c). Of note, $22.8 \%$ of the upregulated genes were TCF1 direct targets in our analysis of a previously reported ChIP-seq dataset ${ }^{43}$, in comparison to $10.0 \%$ of genome bound by TCF1 (Fig. 6b) ${ }^{43}$, reflecting an enrichment of TCF1modulated genes among the upregulated genes. Gene-set enrichment analysis (GSEA) revealed that a memory $\mathrm{CD}^{+} \mathrm{T}$ cell signature was enriched in the LSD1-deficient CD8 ${ }^{+}$TILs (Fig. 6c). A number of genes, for instance Il7r, Slamf6, and Bcl6, which are TCF1 targets and associated with the progenitor phenotype of CD $8^{+}$TILs $^{6,43}$, were significantly upregulated by LSD1 depletion (Fig. 6a). Interestingly, the expression of a few cytokines, including TNFa, LTA, XCL1, CCL3, and CCL4, was also induced in the LSD1-deficient CD8 ${ }^{+}$TILs, while several murinespecific granzymes (granzyme C, D, E, F, and G) of unknown activity were decreased (Fig. $6 \mathrm{a})^{44}$. Consistent with the resistance to apoptosis (Fig. 4m, n), Bcl2l11 encoding pro-apoptotic BIM was reduced in the LSD1-deficient CD8 ${ }^{+}$TILs (Fig. 6a).

To further confirm the RNA-seq data, we analyzed gene expression by flow cytometry in the MC38 tumor model. Indeed, intratumoral CD8 ${ }^{+}$T cells without LSD1 showed increased IL7Ra and Slamf6 (Fig. 6d-g). These phenotypes were consistently recapitulated in the TRAMP-C2 tumor model (Supplementary Fig. $11 \mathrm{a}-\mathrm{d})$. In line with the progenitor phenotype of $\mathrm{CD} 8{ }^{+} \mathrm{PD}-$ $1^{\text {int }} \mathrm{T}$ cells, this population expressed higher levels of IL7R $a$ and Slamf6 than did the $\mathrm{CD} 8^{+} \mathrm{PD}-1^{\text {hi }} \mathrm{T}$ cells (Supplementary Fig. 11e-j). Notably, LSD1 loss significantly upregulated IL7Ra and Slamf6 in the CD8 ${ }^{+} \mathrm{PD}-1^{\text {int }} \mathrm{T}$ cells, in contrast to a mild effect in the $\mathrm{CD}^{+} \mathrm{PD}-1^{\text {hi }} \mathrm{T}$ cells (Supplementary Fig. 11e-j), further supporting the functional significance of the proposed interplay between LSD1 and TCF1 in the progenitor exhausted CD8 ${ }^{+}$TILs. In line with the decreased TOX expression observed by RNA-seq analysis (Fig. 6a), LSD1 depletion compromised the differentiation of $\mathrm{TCF}^{+}$cells into a more exhausted state marked by TOX expression (Supplementary Fig. 11k-m) ${ }^{23-25}$. The gene expression data thus suggest that TCF1-controlled transcription network involved in $\mathrm{CD}^{+} \mathrm{T}$ cell survival and self-renewal is augmented in response to LSD1 depletion.

Targeting LSD1 promotes long-lasting responses to anti-PD1 treatment. Among exhausted $\mathrm{CD}^{+} \mathrm{T}$ cells, the intratumoral $\mathrm{TCF} 1^{+} \mathrm{PD}-1^{\text {int }}$ progenitor subset has been reported to be the prominent determinant of effective responses to PD-1 blockade ${ }^{6,8,9,13}$. The remarkable effect of LSD1 depletion on sustaining the progenitor exhausted $\mathrm{CD}^{+}$TILs prompted us to examine whether targeting LSD1 could improve tumor responses to anti-PD-1 treatment. Since tumor antigen-driven $\mathrm{T}$ cell exhaustion has been suggested to occur early in tumor development ${ }^{45}$, we initiated GSK2879552 treatment on the second day after tumor implantation, followed by anti-PD1 treatment two weeks later when tumors were well-established (Fig. 7a). The growth of MC38 tumors was significantly restrained by either GSK2879552 or anti-PD-1 treatment, and the combination of these two treatments displayed a cooperative effect on controlling tumor growth (Fig. 7b). Notably, tumors primed with GSK2879552 treatment mostly showed prolonged responses to PD-1 blockade with a significant increase of tumor rejection rate $(80 \%, 12 / 15$ versus $47 \%$, 7/15) (Fig. 7c). Thus, systemic treatment with an LSD1 inhibitor enables long-lasting responses to PD-1 blockade.

To elucidate the mechanisms underlying the long-lasting response of GSK2879552-primed tumors to PD-1 blockade, we conducted comparative analyses of tumor immune microenvironment in response to different treatment. GSK2879552 and/or anti-PD1 treatment had no overt effect on the infiltration of MDSCs, DCs or NK cells, but GSK2879552 exerted a suppressive effect on GzmB expression by NK cells (Supplementary Fig. 12a-d). Similar to the results from the genetic study (Figs. $2 \mathrm{~h}$ and 4e), GSK2879552 significantly increased the pool size of the $\mathrm{TCF} 1^{+} \mathrm{PD}-1^{\text {int }}$ progenitor exhausted CD8 ${ }^{+}$TILs (Fig. 7d), but did not affect the overall GzmB expression by $\mathrm{CD}^{+}$TILs (Fig. 7e). Upon anti-PD-1 treatment, 
a

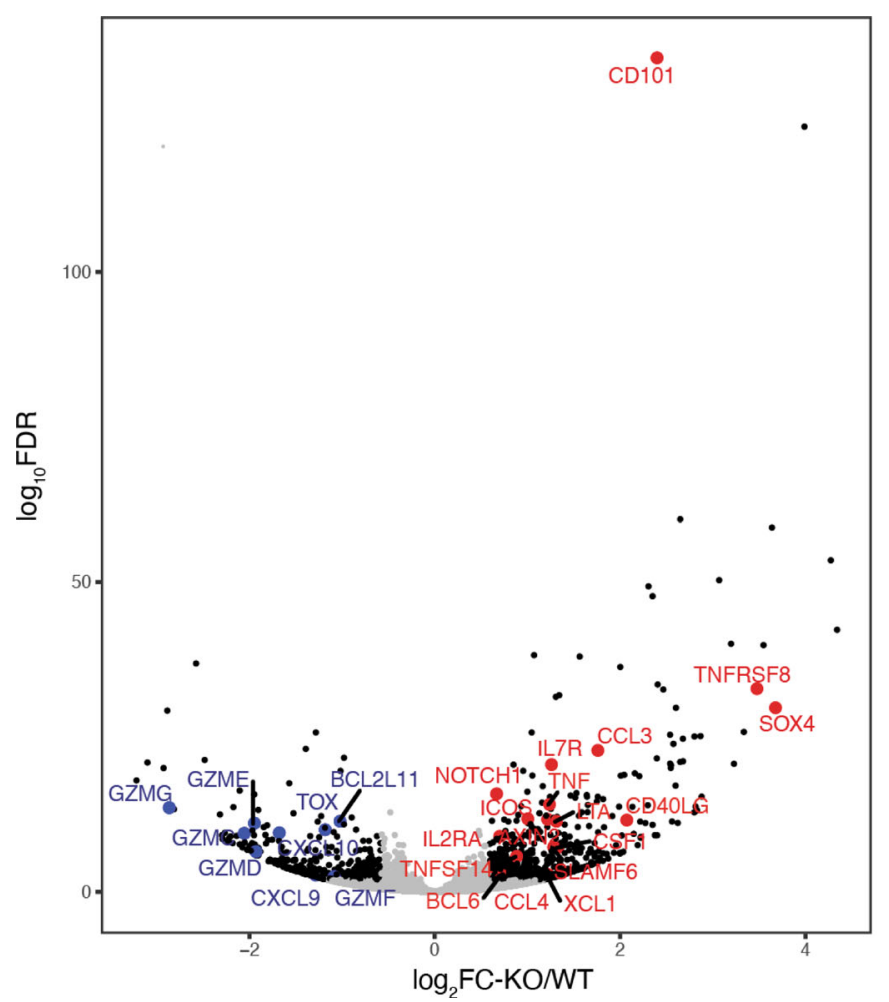

b

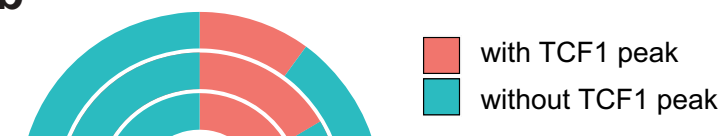

From inside to outside:

- up-regulated genes

- down-regulated genes

- all genes
$\mathrm{TSS} \pm 2 \mathrm{k}$

C
GSE41867_memory VS exhausted CD8 T cell

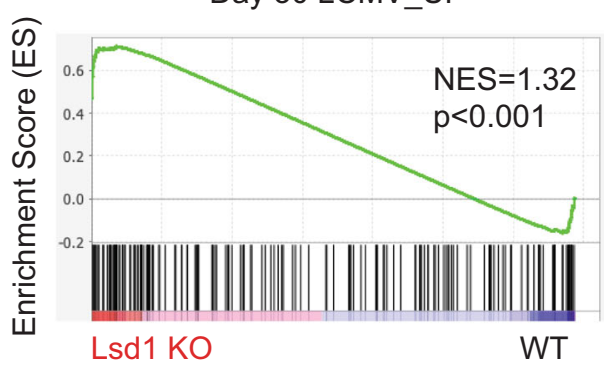

d Gated on CD8 ${ }^{+}$PD1 $1^{+}$TLL

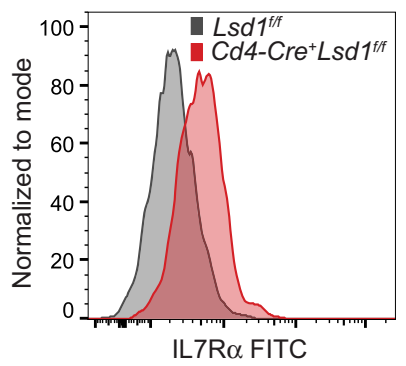

e

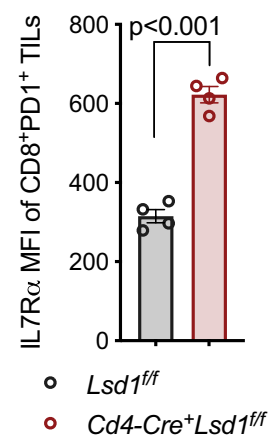

f

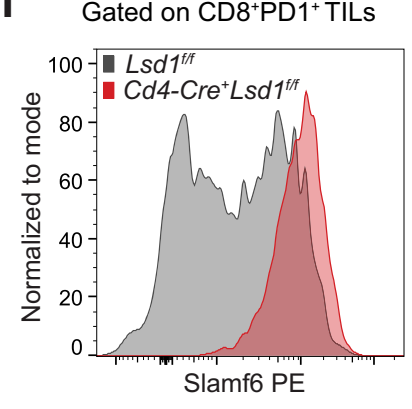

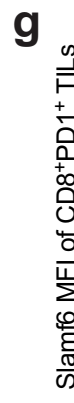

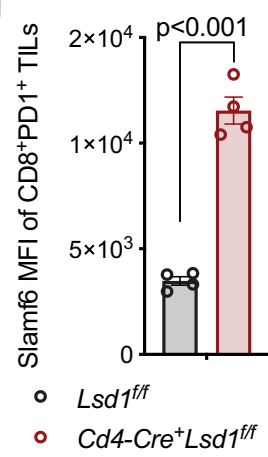

Fig. 6 LSD1 loss unleashes TCF1-controlled gene expression in CD8 ${ }^{+}$TILs. a A volcano plot showing differential gene expression by LSD1-deficient versus wildtype $\mathrm{CD} 8{ }^{+} \mathrm{CD} 44^{+} \mathrm{PD}-1^{+}$TILs of MC38 tumors ( $n=4$ per group, FC $>1.5$ and FDR $<0.01$ as the cutoff). Non-gray colored dots mean significant difference. $\mathbf{b}$ Percentages of genes bound by TCF1 among the differentially expressed genes in LSD1-deficient versus wildtype CD8 ${ }^{+}$TILs or among total genes in genome. c GSEA analysis in Lsd1 KO versus WT CD8 ${ }^{+}$TILs ( $n=4$ per group). Gene list was ranked with signed (from log $\left.{ }_{2} \mathrm{FC}\right)$ likelihood ratio from Lsd1 KO versus WT comparison. Kolmogorov-Smirnov test was used to determine the enrichment score. d, e A representative flow plot of IL7R $\alpha$ expression (d) and IL7R $\alpha \mathrm{MFI}(\mathbf{e})$ of MC38 tumor-infiltrating CD8 ${ }^{+}$PD- $1^{+}$T cells ( $n=4$ per group). $\mathbf{f}, \mathbf{g}$ A representative flow plot of Slamf6 expression ( $\mathbf{f}$ ) and Slamf6 MFI (g) of MC38 tumor-infiltrating CD8+PD-1+ T cells $(n=4$ per group). Data represent two independent experiments (d-g) and are presented as mean $\pm \operatorname{SEM}(\mathbf{e}, \mathbf{g})$. Statistical significance was determined by two-sided unpaired $t$ test $(\mathbf{e}, \mathbf{g})$. Source data are provided as a Source data file.

GzmB expression by CD8 ${ }^{+}$TILs was strongly increased independent of prior GSK2879552 treatment (Fig. 7e). In fact, the frequency of GzmB-expressing CD8 ${ }^{+}$TILs was comparable in tumors treated with anti-PD-1 alone or together with GSK2879552 (Fig. 7e). Under this treatment regimen (Fig. 7a), anti-PD-1 treatment alone did not have an overt effect on the infiltration of $\mathrm{CD}^{+}$or $\mathrm{CD}^{+}$TILs, whereas it significantly and preferentially expanded intratumoral $\mathrm{CD}^{+} \mathrm{T}$ cells only when tumors were primed with GSK2879552 treatment (Fig. 7f, g and Supplementary Fig. 12e). In addition, GSK2879552 treatment increased the generation of the TCF1-TIM$3^{+}$terminally exhausted CD8 ${ }^{+}$TILs upon PD-1 blockade (Fig. 7h, i), likely through promoting their conversion from a larger pool of the TCF $1^{+} \mathrm{PD}-1^{1}{ }^{\text {int }}$ progenitor exhausted CD8 ${ }^{+}$TILs (Fig. 7d) ${ }^{6,8,9,11}$. Taken together, these data suggest that LSD1 inhibition preserves the progenitor exhausted $\mathrm{CD} 8^{+}$TILs and sustains intratumoral $\mathrm{T}$ cell expansion, resulting in long-lasting responses of tumors to anti-PD-1 treatment.

\section{Discussion}

The ineffective reinvigoration of exhausted $\mathrm{T}$ cells accounts for the lack of durable responses to PD-1 blockade therapy. Among 
a

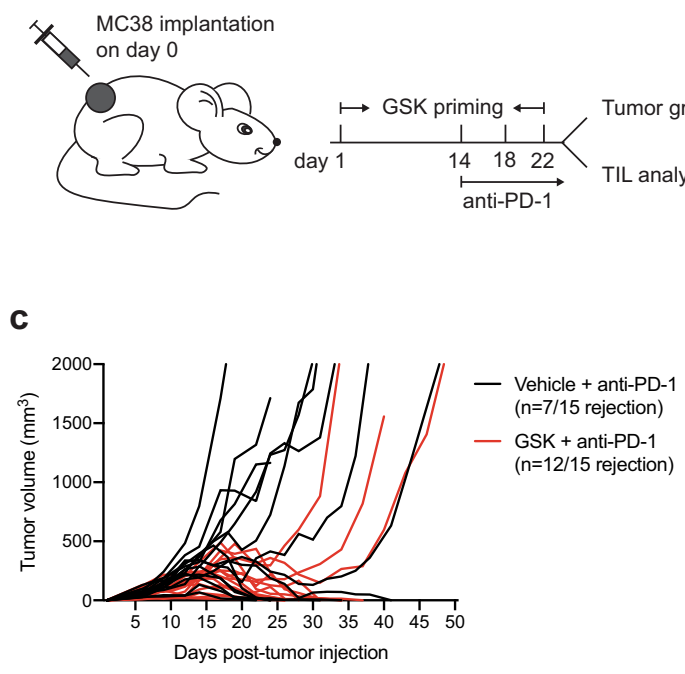

b

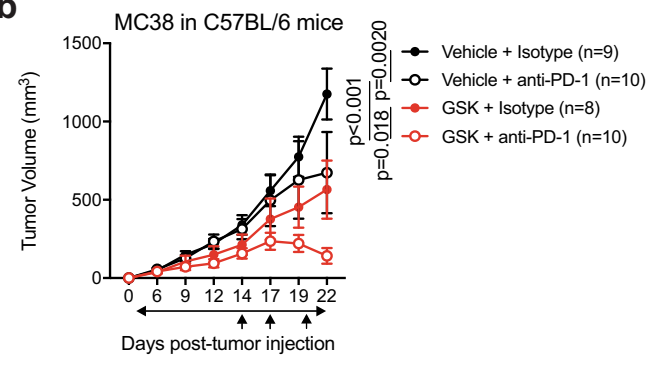

e

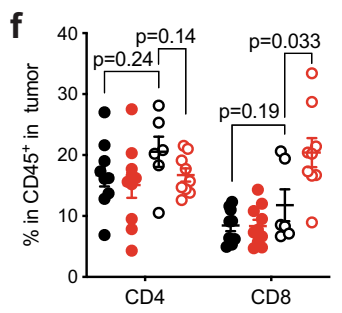

d

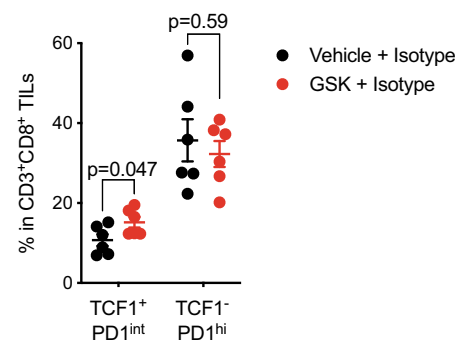

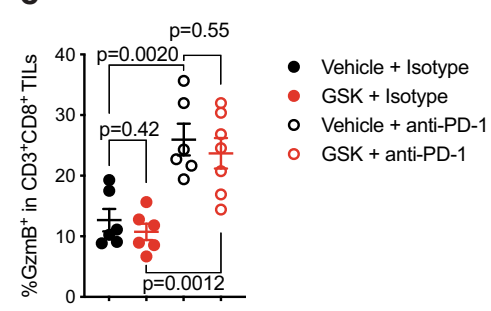

Fig. 7 Targeting LSD1 enhances long-lasting response to anti-PD-1 treatment. a Experimental design of administering GSK2879552 and PD-1 blocking antibodies. b Tumor growth curves of wildtype mice inoculated with MC38 tumor cells and treated with GSK2879552 or vehicle control in combination with anti-PD-1 or isotype control. c Individual tumor growth curves over time in response to anti-PD1 treatment alone or in combination with GSK2879552. d, e Percentages of TCF1+PD-1 $1^{\text {int }}$ and TCF1-PD-1hi cells (d) or GzmB + cells (e) in CD8 ${ }^{+}$TILs (Vehicle + Isotype, $n=6$; GSK + Isotype, $n=6$;

Vehicle + anti-PD-1, $n=6$; GSK + anti-PD-1, $n=7)$. f, g, Percentages $(\mathbf{f}, n=9,10,6,9$, respectively) and cell numbers $(\mathbf{g}, n=8,9,6,5$, respectively) of tumor-infiltrating $\mathrm{CD}^{+}{ }^{+} \mathrm{CD} 4^{+}$T cells and $\mathrm{CD} 3^{+} \mathrm{CD} 8^{+}$T cells in gated $\mathrm{CD} 45^{+}$leukocytes. $\mathbf{h}$, i Frequencies $(\mathbf{h}, n=9,10,6,9$, respectively) and cell numbers $(\mathbf{i}, n=8,9,6,5$, respectively) of CD8+TCF1-TIM-3+ TILs. Data represent two independent experiments $(\mathbf{b}, \mathbf{d}-\mathbf{i})$ or are pooled from two independent experiments (c), and are presented as mean $\pm \operatorname{SEM}(\mathbf{b}, \mathbf{d}-\mathbf{i})$. Sample sizes are as indicated. Statistical significance was determined by two-way ANOVA (b) or two-sided unpaired $t$ test $(\mathbf{d}-\mathbf{i})$. Source data are provided as a Source data file.

heterogeneous and exhausted antigen-specific $\mathrm{CD}^{+} \mathrm{T}$ cells in chronic viral infections and cancer, the $\mathrm{TCF}^{+}$progenitor cells preferentially respond to PD-1 blockade ${ }^{6-9,11}$. Nevertheless, proliferation of the progenitor cells driven by antigen stimulation progressively induces their conversion to terminally differentiated cells, even when PD-1 pathway is blocked. Although not formally proven experimentally, this represents a plausible reason for the transient response to anti-PD-1 treatment ${ }^{2}$. Thus, maintaining and/or expanding such progenitor $\mathrm{CD} 8{ }^{+} \mathrm{T}$ cell population in the TME may lead to a long-lasting tumor response to anti-PD-1 treatment. Both our genetic and chemical biology approaches support the notion that LSD1 enforces an epigenetic program, which restrains the progenitor pool and promotes $\mathrm{T}$ cell terminal exhaustion in the TME, possibly through antagonizing TCF1mediated transcription. Therefore, manipulating LSD1 in T cells results in a long-lasting response of tumors to anti-PD1 therapy.

Epigenetic regulation is critically involved in almost all aspects of $\mathrm{T}$ cell biology including lineage commitment, development, activation, differentiation, and memory formation ${ }^{46}$. The role of LSD1 in those biological processes remains largely unexplored. We used CD4-Cre in order to efficiently delete floxed Lsd 1 alleles in TCR $\alpha \beta^{+} \mathrm{T}$ cells, and a noticeable loss of LSD1 protein was observed as early as the CD4 or CD8 single positive (SP) stage, although the CD4-driven Cre recombinase is expected to be expressed earlier at the CD4/CD8 double positive (DP) stage $\mathrm{s}^{47}$. Since LSD1 protein in the DP thymocytes of Cd4-Cre ${ }^{+} L s d 1^{f / f}$ mice was detected at a similar level as the wildtype counterpart, these data suggest that key events specific to DP thymocytes, such as TCRa rearrangements and positive selection, are unlikely affected in $\mathrm{Cd} 4-\mathrm{Cr} e^{+} L s d 1^{f / f}$ mice. Consistently, DP thymocytes remain mostly unaltered upon $L s d 1$ deletion. These observations suggest that the improved antitumor immunity in $\mathrm{Cd} 4-\mathrm{Cr} e^{+}$ $L s d 1^{f / f}$ mice is likely due to a role of LSD1 in regulating the activation and differentiation of mature $\mathrm{T}$ cells upon tumor antigen stimulation, which is further supported by our chemical approach with the LSD1 inhibitors.

Persistent antigen stimulation is the key factor driving $\mathrm{CD} 8^{+} \mathrm{T}$ cell exhaustion. In the immunogenic MC38 tumor model, the pool size of the progenitor CD8 ${ }^{+}$TILs was small $(<8 \%)$, so the long-term antitumor immunity was likely limited by the maintenance of the progenitor subset of exhausted CD8 ${ }^{+}$TILs, which can be significantly improved by perturbing T cell-intrinsic LSD1. In contrast, in poorly immunogenic B16 melanoma model, a substantial portion $(>30 \%)$ of CD8 ${ }^{+}$TILs showed the progenitor phenotype, which was further elevated close to $\sim 60 \%$ by the LSD 1 depletion. Thus, a majority of LSD1-deficient $\mathrm{CD}^{+}$TILs retained the progenitor phenotype, thereby compromising the timely conversion to terminally exhausted CD8 ${ }^{+}$TILs that are 
responsible for the immediate tumor-killing effect and short-term tumor control. As a result, B16 tumors displayed an accelerated outgrowth shortly after implantation in T cell-specific LSD1 knockout mice. Hence, perturbation of $\mathrm{T}$ cell-intrinsic LSD1 consistently increases the progenitor subset of exhausted CD8 ${ }^{+} \mathrm{T}$ cell in the TME in various tumor models, but drives tumor outgrowth differently depending on tumor context. The impact of targeting T cell-intrinsic LSD1 on B16 tumor growth is opposite to our previous finding that the ablation of tumor cell-intrinsic LSD1 enhances tumor immunogenicity, suppresses tumor growth and overcomes tumor primary resistance to PD1 blockade therapy ${ }^{31}$. This highlights the importance of tumor stratification as well as targeted drug delivery approach for reaching a combinatory antitumor effect of LSD1 inhibitors with PD1 blocking antibodies.

$\mathrm{T}$ cell activation, differentiation, and exhaustion, driven by tumor antigens-induced chronic stimulation and regulated by tumor microenvironmental factors, display significant remodeling of the chromatin landscapes ${ }^{6,16,18}$. The importance of chromatin regulation in such biological processes has been well recognized, but how it actually works to influence $\mathrm{T}$ cell activation and differentiation remains incompletely understood. Our study uncovers a critical role of the histone demethylase LSD1 in controlling the balance between the progenitor exhausted and the terminally exhausted $\mathrm{CD}^{+}$TILs. The observation that LSD1 only interacted with the long but not short isoforms of TCF1 highlights an important role of LSD1 in the progenitor exhausted CD8 ${ }^{+}$TILs, since the long isoform of TCF1 has been suggested to sustain the stem-like properties ${ }^{8,48,49}$, which is likely to be suppressed by LSD1 through their interaction. Once the progenitor exhausted CD8 ${ }^{+}$TILs lose the expression of TCF1 long isoform and subsequently convert to terminally exhausted cells, LSD1 may no longer have substantial effects. Indeed, we found apoptosis of the terminally exhausted cells largely unaffected by LSD1 loss, in contrast to the reduced apoptosis in the progenitor exhausted cells. The presence of $\beta$-catenin binding domain in the long isoform of TCF1 could mediate the recruitment of $\beta$-catenin to the interacting protein complex, which was detected in the TCF1 p45 as well as LSD1 immunoprecipitates. However, it remains to be determined whether the interaction with $\beta$-catenin is necessary for the function of the long isoform of TCF1, particularly given a long-standing debate on the necessity of $\beta$-catenin in memory $\mathrm{T}$ cell formation ${ }^{50-52}$. Alternatively, other co-factors, such as ATF2 ${ }^{53}$, may also bind to and regulate the transcriptional activity of the long isoform of TCF1 independent of the canonical Wnt- $\beta$-catenin signaling.

The physical interaction of LSD1/CoREST complex with TCF1 is consistent with the idea that LSD1 needs other components of this complex to mediate gene repression $38,54,55$, and supports the involvement of other components of the complex in regulating $\mathrm{CD}^{+} \mathrm{T}$ cell exhaustion. Since LSD1 also mediates demethylation of non-histone proteins ${ }^{37}$, whether LSD1 influences the function of TCF1 via demethylation of TCF1 or its binding partners remains to be determined. Collectively, understanding the precise biochemical mechanism of how LSD1 interacts with TCF1 to impact its transcription program requires further investigations in the progenitor exhausted CD8 ${ }^{+} \mathrm{T}$ cells. Additionally, future investigations of histone modifications, including methylation and acetylation, in different subsets of $\mathrm{CD} 8^{+}$TILs will provide more in-depth insights into the chromatin mechanisms underlying the maintenance and differentiation of the progenitor CD8 ${ }^{+}$TILs.

LSD1 is upregulated in a variety of cancers and mostly plays a tumor-promoting role through multiple mechanisms including maintaining the stemness of tumor cells ${ }^{56-58}$, supporting tumor cell proliferation ${ }^{30,59}$, and suppressing antitumor immunity ${ }^{31}$.
Thus, LSD1 has been actively explored as a target for cancer treatment. Notably, LSD1 also plays critical physiological roles in haematopoiesis ${ }^{60}$ as well as neuronal differentiation and function $^{61-63}$, and the administration of some irreversible LSD1 inhibitors has been found to cause adverse toxicities in some cancer clinical trials ${ }^{64}$. In contrast, some other irreversible LSD1 inhibitors and also recently developed reversible LSD1 inhibitors tested appear to have no dose-limiting toxicities in recent clinical studies ${ }^{64}$. The crucial role of LSD1 in $\mathrm{CD}^{+} \mathrm{T}$ exhaustion that we identify and report here warrants further consideration of LSD1 inhibition in combination with PD-1 pathway inhibitors in cancer therapy. In one approach, current LSD1 inhibitors with tolerable toxicities could be explored to prime $\mathrm{CD}^{+} \mathrm{T}$ cells for a short duration to improve their sustainable responses to PD-1 blockade. In another approach, based on the unique mechanism of action of LSD1 in intratumoral $\mathrm{CD} 8^{+} \mathrm{T}$ cells mediated by its interaction with TCF1 long isoform, we propose that antagonists, either small molecules or biologics that disrupt the interaction between LSD1 and the long isoform TCF1, could be developed in cancer checkpoint blockade therapy. Moreover, both approaches of inhibiting LSD1 or genetic ablation of LSD1 could also be exploited to enhance the persistence of cytotoxic $T$ cells in adoptive T-cell therapy for cancer. Further studies are warranted to stratify tumors that may benefit from the combination of LSD1 perturbation with immunotherapy.

In summary, our study uncovers an important role of LSD1 and its mechanism of action in regulating intratumoral $\mathrm{CD}^{+}$ $\mathrm{T}$ cells and its impact on tumor growth control. Our study also highlights the translational significance of combining LSD1 inhibitors with PD-1 blockade in cancer treatment, in which LSD1 inhibition in $\mathrm{CD}^{+} \mathrm{T}$ cells potentiates the progenitor phenotype and thus promotes long-lasting responses to $\mathrm{PD}-1$ blockade. Our current data suggest that certain tumors respond to the combination of LSD1 inhibition and PD-1 blockade, thus identification of biomarkers is necessary to help stratify patients in order to significantly enhance the clinical development and benefits of this approach. The identification of a specific mechanism of action of LSD1 in intratumoral CD8 ${ }^{+} \mathrm{T}$ cells also points to a promising avenue for targeting LSD1 in cancer immunotherapy through specifically disrupting the physical interaction between LSD1 and the long isoform of TCF1, a pivotal regulator driving the progenitor phenotype maintenance.

\section{Methods}

Cell culture. MC38, B16, and HEK293T cells were cultured in DMEM medium containing $10 \%$ heat-inactivated FBS and $1 \%$ penicillin/streptomycin in a $5 \% \mathrm{CO}_{2}$ incubator at $37^{\circ} \mathrm{C}$. TRAMP-C2 cells were cultured in DMEM medium supplemented with $5 \% \mathrm{Nu}$-Serum IV, $5 \%$ heat-inactivated FBS, $5 \mu \mathrm{g} / \mathrm{ml}$ bovine insulin, 10 nM DHEA and $1 \%$ penicillin/streptomycin in a $5 \% \mathrm{CO}_{2}$ incubator at $37^{\circ} \mathrm{C}$. Isolated CD8 ${ }^{+} \mathrm{T}$ cells were cultured in R10 medium (RPMI 1640 supplemented with $10 \%$ FBS, $1 \%$ penicillin/ streptomycin, $12 \mathrm{mM}$ HEPES and $50 \mu \mathrm{M} 2$-mercaptoethanol). MC38 cell line was a gift from Dr. Arlene Sharpe and B16/F10 was a gift from Dr. David Fisher. Both cell lines were originally purchased from ATCC. MC38-OVA cell line was a gift from Dr. Ana Anderson and B16-OVA cell line was gift from Dr. Nick Haining. HEK293T and TRAMP-C2 cell line were purchased from ATCC.

Mice. Six to ten-week-old mice were used for all experiments. Wildtype C57BL/6 mice were purchased from The Jackson Laboratory. Cd4-Cre transgenic mice (purchased from The Jackson Laboratory, stock \#017336) were crossed with Lsd1 floxed mice (gifts from Dr. Stuart Orkin at Boston Children's Hospital) to generate $C d 4-C r e^{+} L s d 1^{f / f}$ knockout mice and Lsd1 $1^{f / f}$ littermate control mice. Immunodeficient TCRa knockout mice were originally purchased from The Jackson Laboratory (stock \#002116) and bred in-house. CD45.1 mice were purchased from The Jackson Laboratory (stock \#002014). Prior to all experiments, purchased mice were allowed to acclimate to housing conditions at the Boston Children's Hospital Animal Facility for one week. Mice were subcutaneously inoculated with tumor cells and were euthanized when tumor volumes exceed 2000 cubic millimeters. All experimental mice were housed in specific pathogen-free conditions and all animal procedures were performed in accordance with animal care guidelines and with the 
prior approval by the Boston Children's Hospital Institutional Animal Care and Use Committee. Male animals were used for TRAMP-C2 related experiments. Female animals were mostly used for other experiments.

Mouse subcutaneous tumor models. $2.5 \times 10^{5}$ or $5 \times 10^{5} \mathrm{MC} 38$ or B16, or $10^{6}$ TRAMP-C2 cells in $1 \times$ PBS were subcutaneously injected into the right hind flank of each pre-shaved wildtype, $C d 4-C r e^{+} L s d 1^{f / f}$ or $L s d 1^{f / f}$ mouse to establish tumors. Both male and female mice were used in MC38 and B16 tumor models and only male mice were used in TRAMP-C2 tumor model. Tumors were manually measured and recorded using a digital caliper starting from day 6 every 2-3 days. Tumor size was calculated using the following formula: $1 / 2 \times$ length $\times$ width $^{2}$. Mice reached the endpoint when tumor volumes were over $2000 \mathrm{~mm}^{3}$.

For T cell reconstitution, $\mathrm{CD}^{+} \mathrm{T}$ cells were isolated from spleens of $\mathrm{Cd} 4-\mathrm{Cr} e^{+}$ $L s d l^{f / f}$ or $L s d 1^{f / f}$ mice by magnetic separation using CD8a (Ly-2) microbeads (Miltenyi Biotec, cat\#130-117-044) according to the instruction manual. The isolated $\mathrm{CD}^{+} \mathrm{T}$ cells were resuspended in $1 \times \mathrm{PBS}$ and intravenously transferred into TCRa knockout mice at 8 million cells per mouse. On the next day, reconstituted mice were inoculated with $2.5 \times 10^{5} \mathrm{MC} 38$ cells for tumor growth and TIL analysis experiments.

For drug treatments, mice were administered with rat IgG2a isotype control (BioXCell, clone 2A3), anti-PD-1 (BioXCell, clone 29F.1A12), vehicle or GSK2879552 (MedChem Express, cat\#HY-18632) at the time points shown in the figures. $100 \mu \mathrm{g}$ anti-PD-1 or isotype control were injected intraperitoneally into each mouse every 3 days as indicated in the figures. GSK2879552 or vehicle was injected intraperitoneally into each mouse every day after tumor inoculation at a dose of $\sim 1.5 \mathrm{mg} / \mathrm{kg}$ body weight. Prior to treatments, mice were randomized such that treatment groups had similar average tumor volumes prior to treatment initiation. FTY720 treatment was initiated at day 12 post-tumor inoculation at a dose of $1 \mathrm{mg} / \mathrm{kg}$ body weight and followed by intraperitoneal injection at a dose of $0.2 \mathrm{mg} / \mathrm{kg}$ daily until tumor collection.

Isolation and in vitro activation of splenic $\mathrm{CDB}^{+} \mathbf{T}$ cells. Spleens were harvested from $C d 4-C r e^{+} L s d 1^{f f f}$ and $L s d 1^{f f f}$ mice and $\mathrm{CD}^{+} \mathrm{T}$ cells were isolated by magnetic separation using CD8a (Ly-2) microbeads (Miltenyi Biotec, cat\#130-117-044) according to the instruction manual. The isolated $\mathrm{CD} 8^{+} \mathrm{T}$ cells were resuspended in R10 medium at $5 \times 10^{5} / \mathrm{ml}$ and plated onto 24 -well plates pre-coated with $3 \mu \mathrm{g} / \mathrm{ml}$ anti-CD3 (BioLegend, cat\#100340), supplemented with $2 \mu \mathrm{g} / \mathrm{ml}$ anti-CD28 (BioLegend, cat\#102116) and $25 \mathrm{ng} / \mathrm{ml} \mathrm{IL-2} \mathrm{(PeproTech,} \mathrm{cat \# 200-02).} \mathrm{CD8}{ }^{+} \mathrm{T}$ cells were treated with GSK2879552, iCRT3 (MedChem Express, cat\#HY-103705) or vehicle as indicated. After $72 \mathrm{~h}$ of in vitro stimulation, activated $\mathrm{CD}^{+} \mathrm{T}$ cells were collected, stained with antibodies against surface markers, and when needed, fixed and permeabilized with a Foxp3/Transcription Factor Staining Buffer Set (eBioscience, cat\#00-5523-00), followed by intracellular staining. 7-AAD Viability Staining Solution (BioLegend, cat\#420404) was added lastly to exclude dead cells for unfixed cells, and LIVE/DEAD ${ }^{\mathrm{m}}$ Fixable Near-IR Dead Cell Stain Kit (Thermo Fisher Scientific, cat\#L10119) was used for cells subjected to fixation. Flow cytometry data were acquired on a BD LSR II or BD FACSymphony using FACSDiva v8.0.1 and analyzed by FlowJo 10.4 software.

Tumor-infiltrating leukocyte analysis by flow cytometry. $5 \times 10^{5} \mathrm{MC} 38$ or B16, or $10^{6}$ TRAMP-C2 cells were subcutaneously injected into the right flank of individual mouse and tumors were harvested on day $12-20$ as indicated after tumor inoculation. Tumors were minced into small pieces and digested in RPMI1640 medium containing $400 \mathrm{U} / \mathrm{ml}$ type I collagenase (Worthington Biochemical Corporation, cat\#LS004194) and $100 \mu \mathrm{g} / \mathrm{ml}$ DNase I (Sigma-Aldrich, cat\#10104159001) for $20-30 \mathrm{~min}$ at $37^{\circ} \mathrm{C}$. Digested tumor tissue samples were neutralized with R10 medium and then filtered through a $70 \mu \mathrm{M}$ cell strainer to obtain single cell suspensions. Samples were pelleted and resuspended in $5 \mathrm{ml}$ of $40 \%$ Percoll (GE Healthcare, cat\#17-0891-01) and underlayed by $3 \mathrm{ml}$ of $70 \%$ Percoll in a $15 \mathrm{ml}$ conical tube. After centrifugation at $800 \mathrm{~g}$ for 20 min with break set at 1 , leukocytes were enriched at the interface between 40 and $70 \%$ Percoll gradient. Collected leukocytes from the gradient interface were then resuspended in ACK lysis buffer to remove red blood cells and then stained with antibodies against surface markers and intracellular proteins, or SIINFEKL H-2Kb Tetramer (NIH Tetramer Core Facility) as needed. For cytokine staining, leukocytes were first stimulated with $1 \mu \mathrm{M} \mathrm{OVA}_{257-264}$ peptides (Anaspec, cat\#AS-60193-1) or PMA/ Ionomycin in the presence of Golgiplug for $4 \mathrm{~h}$. Stained tumor-infiltrating leukocyte (TIL) samples were run on a BD LSR II or BD FACSymphony using FACSDiva v8.0.1 and analyzed by FlowJo software. All antibodies were purchased from BioLegend, Thermo Fisher Scientific or BD Biosciences and were used at 1:200 dilution: SIINFEKL H-2K $\mathrm{K}^{\mathrm{b}}$ Tetramer (NIH Tetramer Core Facility); LIVE/DEAD ${ }^{\mathrm{m}}$ Fixable Near-IR Dead Cell Stain Kit (ThermoFisher Scientific, L10119); IgG2a isotype control (clone 2A3, BioXCell, BE0089); anti-PD-1 (BioXCell, clone 29F.1A12, BE0273); CD45.2, BV421 (clone 104, BioLegend, Cat\#109831); CD45.2, PE (clone 104, BioLegend, Cat\#109807); CD45.1, FITC (clone A20, BioLegend, Cat\#110705); CD3e, BV510 (clone145-2C11, BioLegend, Cat\#100353); TCRb, BV510 (clone H57-587, BioLegend, Cat\#109233); CD4, APC (clone RM4-5, BioLegend, Cat\#100516); CD8b, APC/Cy7 (clone YTS156.7.7, BioLegend, Cat\#126619); CD8b, PE (clone YTS156.7.7, BioLegend, Cat\#126607); CD8a, BV605 (clone 53-6.7, BioLegend, Cat\#100743); CD8a, BV510 (clone 53-6.7, BioLegend, Cat\#100751); Foxp3, PE (clone FJK-16s, ThermoFisher Scientific, Cat\#12-5773-82); Granzyme-B, FITC (clone GB11, BioLegend, Cat\#515403); Ki-67, PercCP-Cy5.5 (clone B56, BD Biosciences, Cat\#561284); CD44, FITC (clone IM7, BioLegend, Cat\#103005); CD62L, BV510 (clone MEL-14, BioLegend, Cat\#104441); CD62L, PE (clone MEL-14, BioLegend, Cat\#104407); CD16/32 (clone 93, BioLegend, Cat\#101320); 7-AAD viability staining solution (BioLegend, Cat\#420404); CD11b, BV605 (clone M1/70, BioLegend, Cat\#101237); Gr-1, APC-Cy7 (clone RB6-8C5, BioLegend, Cat\#108423); TNFa, FITC (cloneMP6-XT22, BioLegend, Cat\#506303); IL-2, PerCP-Cy5.5 (cloneJES6-5H4, BioLegend, Cat\#503821); IFN-g, PE (clone XMG1.2, BioLegend, Cat\#104407); PD-1, PE-Cy7 (29F.1A12, BioLegend, Cat\#135215); Tim-3, PE(RMT3-23, BioLegend, Cat\#119703); Tim-3, APC (RMT323, BioLegend, Cat\#119705); TCF1/TCF7, AF647 (C63D9, CST, Cat\#6709S); CD127, PE-Cy7 (A7R34, BioLegend, Cat\#135013); CD127, FITC (A7R34, BioLegend, Cat\#135007); TOX, PE (TXRX10, eBiosciences, Cat\#12-6502-80); Slamf6, PE (clone 13G3, eBiosciences, Cat\#12-1508-80).

CD8 ${ }^{+}$TIL co-transfer assay. Congenic CD45.1 ${ }^{+}$and $C d 4-C r e^{+} L s d l^{f / f}\left(\mathrm{CD} 45.2^{+}\right)$ mice were implanted with $5 \times 10^{5}$ MC38 tumor cells for 14 days, followed by tumor excision and TIL isolation as described above. CD8 ${ }^{+}$TILs were further isolated by magnetic separation using CD8a (Ly-2) microbeads. The number of CD8 ${ }^{+}$TILs was quantified by staining a small aliquot with antibodies against $\mathrm{CD} 45, \mathrm{CD} 3$, and CD8 for flow cytometry. CD45.1 $1^{+} \mathrm{CD}^{+}$wildtype TILs and CD45.2 ${ }^{+} \mathrm{CD} 8{ }^{+}$LSD1deficient TILs were then mixed in equal numbers and intravenously transferred into TCR $\alpha$ knockout recipient mice at 20,000 cells per mouse. On the next day, recipient mice were implanted with $5 \times 10^{5}$ MC38 tumor cells. After 14 days, TILs were isolated as described above and the frequencies of $\mathrm{CD} 45.1^{+}$and $\mathrm{CD} 45.2^{+}$ cells among TCR $\beta^{+} \mathrm{CD} 8^{+}$TILs were analyzed by flow cytometry.

In vivo transfer and persistence assay. 7-week-old female C57BL/6J mice were subcutaneously implanted with $5 \times 10^{5}$ MC38 cells on day 0 . CD8 ${ }^{+}$TILs were isolated from MC38 tumors on day 20 as described above and stained with antibodies against CD45.2 (clone 104), CD3 (clone145-2C11), CD8 (clone YTS156.7.7), PD-1 (clone 29F.1A12) and TIM-3 (clone RMT3-23). 7-AAD Viability Staining Solution was used to exclude dead cells. PD-1 $1^{\text {int Tim-3- }}$ or PD$1^{\text {hiTim-3- CD }}{ }^{+}$TILs were sorted and resuspended in PBS. 10,000 sorted cells of these two populations were transferred via intravenous tail vein injection into TCRa KO mice respectively, which were implanted with $2.5 \times 10^{5}$ MC38 cells on the next day. TILs were isolated after 12-14 days of tumor implantation and analyzed to determine the number and immunological phenotype of the transferred $\mathrm{CD} 8^{+}$cells.

Apoptosis assay. Tumor-infiltrating leukocytes isolated as described above were first stained with antibodies against surface markers in MACS buffer. After washing, cells were resuspended in Annexin V binding buffer and stained with Annexin V FITC (SouthernBiotech, cat\#10010-02). Alternatively, cells in MACS buffer were incubated with CellEvent Caspase-3/7 Green Detection Reagent (Thermo Fisher Scientific, cat $\# \mathrm{C} 10740$ ) for $25 \mathrm{~min}$ at $37^{\circ} \mathrm{C}$, and then incubated with SYTOX AADvanced dead cell stain solution for $5 \mathrm{~min}$ at $37^{\circ} \mathrm{C}$. Stained samples were analyzed with a BD LSR II for Annexin V positive or Caspase-3/7 positive cells.

TCF/LEF luciferase reporter assay. HEK293T cells were transfected with TCF/ LEF firefly luciferase reporter, renilla luciferase control reporter (gifts from Dr. Xi $\mathrm{He}$ at Boston Children's Hospital) and mammalian expression plasmids carrying $L s d 1, T c f 7$ isoforms or $\beta$-catenin. Cells were lysed $48 \mathrm{~h}$ post-transfection and luciferase activity was measured and calculated according to the Dual-Luciferase Reporter Assay System (Promega) instruction manual.

RNA extraction and real-time qPCR. For RNA extraction, cells were lysed by directly adding TRIzol (Life Technologies, cat\#15596018) onto cells after supernatant removal. Total RNA extraction was performed according to the manufacturer's instructions. The extracted RNA was reversely transcribed into cDNA using the PrimeScript ${ }^{\text {tw }}$ RT Reagent Kit (TaKaRa, cat\#RR037B) and used for realtime quantitative PCR (qPCR). SYBR green (Life Technologies, cat\#A25743) and gene specific primers (listed in Supplementary Table 1) were used for PCR amplification and detection on a QuantStudio 3 real-time PCR system (Applied Biosystems). The qPCR data were normalized to Gapdh and presented as fold changes of gene expression in the test sample compared to the control.

Gene deletion by CRISPR/Cas9. The guide RNA (gRNA) oligos targeting mouse $L s d 1$ and $B 2 m$ (sequences listed in Supplementary Table 1) were annealed and cloned into a lenti-CRISPR-v2-Puromycin ${ }^{+}$vector (Addgene, cat\# \#52961), respectively. Lentivirus carrying lenti-CRISPR plasmid was prepared by cotransfecting HEK293T cells with four helper plasmids (pHDM-VSV-G, pHDMtat1b, pHDM-HgPM2, and pRC-CMVRaII), followed by viral supernatant collection after $72 \mathrm{~h}$. To delete $L s d 1$, MC38 cells were transduced with lenti-CRISPR virus with the addition of $8 \mu \mathrm{g} / \mathrm{ml}$ polybrene (Sigma-Aldrich, cat\#H9268), and 
selected with $1 \mu \mathrm{g} / \mathrm{ml}$ puromycin for 2 days. Cells were serially diluted to allow clone generation from single cells. Clones were validated for $L s d 1$ knockout by sequencing of target genomic regions and immunoblotting. To delete B2m, MC38 cells were selected with $1 \mu \mathrm{g} / \mathrm{ml}$ puromycin for 7 days after viral transduction. Cells were then stained with anti-B2m PE (Santa Cruz, cat\#sc-32241 PE) and sorted on a $\mathrm{BD}$ Aria for $\mathrm{B} 2 \mathrm{~m}$-negative cells.

Co-immunoprecipitation. HEK293T cells were transfected with the indicated plasmids carrying HA- or Flag-tagged $L s d 1$ or $T c f 7$ isoforms, and harvested $48 \mathrm{~h}$ post-transfection. Cell pellets were lysed for $20 \mathrm{~min}$ on ice in IP lysis buffer $(50 \mathrm{mM}$ Tris $\mathrm{pH} 7.5,150 \mathrm{mM} \mathrm{NaCl}, 0.1 \% \mathrm{NP}-40,0.1 \%$ Triton X-100, $10 \%$ glycerol) supplemented with Complete EDTA-free protease inhibitor cocktail (Sigma-Aldrich, cat\#5892791001), 1 mM PMSF and PhosSTOP (Sigma-Aldrich, cat\#04906837001). Protein lysates were briefly sonicated to shear chromatin and then cleared by 15 min centrifugation to pellet cell debris. Cleared protein lysates were incubated with anti-HA magnetic beads (Thermo Fisher Scientific, cat\#88837) or anti-FLAG agarose beads (Sigma-Aldrich, cat\#A2220) for $2 \mathrm{~h}$ at $4^{\circ} \mathrm{C}$. Immunoprecipitates were then washed three times with the IP lysis buffer and eluted using three resin volumes of the elution buffer $(0.5 \mu \mathrm{g} / \mathrm{ml}$ FLAG peptide in IP lysis buffer for antiFLAG resin; 1x SDS loading buffer for anti-HA resin).

Immunoblotting analysis. Whole-cell lysates and immunoprecipitated eluents were denatured with SDS loading buffer and boiled for $5 \mathrm{~min}$ at $95^{\circ} \mathrm{C}$ before resolved on SDS-PAGE gels. Proteins were then transferred onto nitrocellulose membrane, probed with the indicated primary antibodies and detected with HRPconjugated secondary antibodies.

RNA-seq sample processing. CD8 ${ }^{+}$TILs were isolated from MC38 tumorbearing $C d 4-C r e^{+} L s d 1^{f / f}$ and $L s d 1^{f / f}$ mice on day 18 as described above. CD8 ${ }^{+}$TILs from two individual tumor-carrying $L s d 1^{f / f}$ mice were combined as a biological replicate, and $\mathrm{CD} 8^{+}$TILs from each individual tumor-carrying $C d 4-C r e^{+} L s d 1^{f / f}$ mouse was used as a biological replicate. $\mathrm{CD} 8{ }^{+}$TILs were stained with antibodies against CD45.2 (clone 104), TCR $\beta$ (clone H57-587), CD8 (clone YTS156.7.7), CD44 (clone IM7) and PD-1 (clone 29F.1A12), and 7-AAD Viability Staining Solution was used to exclude dead cells. The CD45.2 ${ }^{+} \mathrm{TCR} \beta{ }^{+} \mathrm{CD} 8{ }^{+} \mathrm{CD} 44^{+} \mathrm{PD}-1^{+}$ cells were then sorted on a BD Aria. Approximately 10,000-20,000 sorted cells for each biological replicate were directly lysed in $1 \mathrm{ml}$ TRIzol Reagent (Life Technologies, cat\#15596018). After incubation for $5 \mathrm{~min}, 0.2 \mathrm{ml}$ chloroform was added and mixed by inverting the tubes several times. Samples were incubated for 2-3 min and later centrifuged for $15 \mathrm{~min}$ at $12,000 \times g$ at $4{ }^{\circ} \mathrm{C}$. The upper aqueous phase containing the RNA was collected and mixed with an equal volume of $70 \%$ ethanol, which was then loaded into a spin column from a RNeasy Micro Kit (Qiagen, cat\#74004) and subjected to RNA isolation according to the instruction manual. The on-column DNase digestion was conducted to eliminate DNA contamination.

Purified total RNA was quantified by Qubit (Invitrogen) and used to generate rRNA-depleted RNA with a NEBNext ${ }^{\oplus}$ rRNA Depletion Kit (New England Biolabs, cat\#E6310S) according to the manufacturer's instructions. The rRNAdepleted RNA was purified with a RNA Clean \& Concentrator-5 kit (Zymo Research, cat\#R1016) and then used to generate a directional RNA library with a NEBNext $^{\oplus}$ Ultra $^{\text {Txt }}$ II Directional RNA Library Prep Kit for Illumina ${ }^{\circledR}$ (New England Biolabs, cat\#E7760L) and NEBNext ${ }^{\oplus}$ Multiplex Oligos for Illumina ${ }^{\circledR}$ (New England Biolabs, cat\#E7335L) according to the manufacturer's instructions. Library concentrations and quality were assessed on a Bioanalyzer and by qPCR. The library was sequenced at Nextseq 500 (Illumina) to generate reads from pairedends ( $43 \mathrm{bp}+42 \mathrm{bp}$ ). The raw data are deposited at the Gene Expression Omnibus (GEO) under the subseries entry GSE147130.

RNA-seq data analyses and functional interpretations. The software $\mathrm{STAR}^{65}$ (version 2.4.0e) was used to generate genome indices for mouse reference genome (GRCm38/mm10, December 2011), with two particular specifications including gene annotations, i.e., GENCODE ${ }^{66}$ (vM23, September 2019), and exon-exon junctions, i.e., 35 nucleotides used in constructing the splice junctions database. Next, the high quality paired-end RNA-seq reads were aligned to mouse reference genome, and the consequence of alignment served as the input for featureCounts (version 1.5.0 $)^{67}$ to quantify raw read counts for 55,385 annotated genes, including 21,856 protein-coding genes. Moreover, the normalized unit of reads per kilobase per million mapped reads (RPKM) was generated for every gene annotated in GENCODE, in order to fit the primary request of principle component analysis (PCA). The R function prcomp was used to perform PCA by using the genes having $R P K M \geq 1$ in at least two samples, and the returned two vectors were used as coordinates to make a scatter plot in a 2-dimensional plane.

We used R package DESeq ${ }^{68}$ (version 1.14.1) to identify differentially expressed (DEX) genes between WT and KO. The raw read count per gene served as the input for DESeq2. The GC content correction from CQN package was incorporated to DESeq2 ${ }^{69}$. Since four samples were collected in either condition, they were treated as biological replicates to improve the reliability of DEX genes identification. Statistical tests for differential expression were based on a model using the negative binomial distribution. The reported statistical significances were corrected for multiple testing using the Benjamini-Hochberg procedure with a false discovery rate less than 0.01 . In addition, to be called DEX genes we required the fold change $>1.5$. The up- and downregulated genes in $\mathrm{KO}$ condition were separately queried to Gene Ontology Consortium for gene ontology enrichment assessment, including biological process (BP), molecular function (MF), and cellular component (CC). Moreover, we utilized the software GSEA (version $4.0 .3)^{70}$ to survey the statistically significant concordant differences between KO and WT by using the normalized gene RPKM values, and consequently compared the KO upregulated gene sets to "C7: immunologic signatures" to explore any overrepresented functional term. In addition, a list of upregulated genes and a list of downregulated genes with the cutoff set at FC $>1.5$ and FDR $<0.01$ were sorted. The lists of these genes were uploaded onto the online DAVID (https:// david.ncifcrf.gov/summary.jsp) bioinformatics resources to analyze for enriched GO terms under the category of GOTERM_BP_DIRECT.

TCF1 ChIP-seq data analyses. The previously published TCF1 ChIP-seq data on splenic $\mathrm{CD}^{+} \mathrm{T}$ cells were downloaded from GEO under accession number GSE73240, for which call peaks were conducted with MACS v1.4.2 with TCF1 KO cells as control and with a stringent cutoff $\left(\mathrm{FC} \geq 4, p<10^{-5} \text { and } \mathrm{FDR}<0.05\right)^{43}$. 7807 TCF1 binding sites were retrieved and assigned to gene TSS ( $-2 \mathrm{~kb}$ to $2 \mathrm{~kb}$ ).

Statistical analyses. Statistical analyses were performed using GraphPad Prism 8 software and statistical significance was determined by $p<0.05$. An unpaired Student's $t$ test was used for comparisons between two groups and a two-way ANOVA was used for multiple comparisons of tumor growth. For comparing mouse survival curves, a Log-rank (Mantel-Cox) test was used.

Reporting summary. Further information on research design is available in the Nature Research Reporting Summary linked to this article.

\section{Data availability}

The RNA-seq data are deposited at the Gene Expression Omnibus (GEO) under the accession code GSE147130. The previously published ChIP-seq data are accessible with the code GSE73240. The remaining data of this study are available within the Article, Supplementary Information files or Source Data file. Source data are provided with this paper.

Received: 17 May 2020; Accepted: 3 November 2021; Published online: 24 November 2021

\section{References}

1. Ribas, A. \& Wolchok, J. D. Cancer immunotherapy using checkpoint blockade. Science 359, 1350-1355 (2018).

2. Sharpe, A. H. \& Pauken, K. E. The diverse functions of the PD1 inhibitory pathway. Nat. Rev. Immunol. 18, 153-167 (2018).

3. Sharma, P., Hu-Lieskovan, S., Wargo, J. A. \& Ribas, A. Primary, adaptive, and acquired resistance to cancer immunotherapy. Cell 168, 707-723 (2017).

4. McLane, L. M., Abdel-Hakeem, M. S. \& Wherry, E. J. CD8 T cell exhaustion during chronic viral infection and cancer. Annu Rev. Immunol. 37, 457-495 (2019).

5. Zou, W., Wolchok, J. D. \& Chen, L. PD-L1 (B7-H1) and PD-1 pathway blockade for cancer therapy: Mechanisms, response biomarkers, and combinations. Sci. Transl. Med. 8, 328rv324 (2016).

6. Miller, B. C. et al. Subsets of exhausted CD8(+) T cells differentially mediate tumor control and respond to checkpoint blockade. Nat. Immunol. 20, 326-336 (2019).

7. Sade-Feldman, M. et al. Defining T cell states associated with response to checkpoint immunotherapy in melanoma. Cell 175, 998-1013.e1020 (2018).

8. Siddiqui, I. et al. Intratumoral Tcf1(+)PD-1(+)CD8(+) T cells with stem-like properties promote tumor control in response to vaccination and checkpoint blockade immunotherapy. Immunity 50, 195-211 e110 (2019).

9. Blackburn, S. D., Shin, H., Freeman, G. J. \& Wherry, E. J. Selective expansion of a subset of exhausted CD8 T cells by alphaPD-L1 blockade. Proc. Natl Acad. Sci. USA 105, 15016-15021 (2008).

10. Paley, M. A. et al. Progenitor and terminal subsets of CD8+ T cells cooperate to contain chronic viral infection. Science 338, 1220-1225 (2012).

11. Utzschneider, D. T. et al. T cell factor 1-expressing memory-like CD8(+) $\mathrm{T}$ cells sustain the immune response to chronic viral infections. Immunity 45, 415-427 (2016).

12. He, R. et al. Follicular CXCR5- expressing CD8(+) T cells curtail chronic viral infection. Nature 537, 412-428 (2016).

13. Im, S. J. et al. Defining CD8+ T cells that provide the proliferative burst after PD-1 therapy. Nature 537, 417-421 (2016).

14. Sen, D. R. et al. The epigenetic landscape of T cell exhaustion. Science 354, 1165-1169 (2016) 
15. Pauken, K. E. et al. Epigenetic stability of exhausted T cells limits durability of reinvigoration by PD-1 blockade. Science 354, 1160-1165 (2016).

16. Philip, M. et al. Chromatin states define tumour-specific T cell dysfunction and reprogramming. Nature 545, 452-456 (2017).

17. Mognol, G. P. et al. Exhaustion-associated regulatory regions in CD8(+) tumor-infiltrating T cells. Proc. Natl Acad. Sci. USA 114, E2776-E2785 (2017).

18. Ghoneim, H. E. et al. De novo epigenetic programs inhibit PD-1 blockademediated T cell rejuvenation. Cell 170, 142-157 e119 (2017).

19. Gray, S. M., Amezquita, R. A., Guan, T., Kleinstein, S. H. \& Kaech, S. M. Polycomb repressive complex 2-mediated chromatin repression guides effector CD8(+) T cell terminal differentiation and loss of multipotency. Immunity 46, 596-608 (2017).

20. He, S. et al. Ezh2 phosphorylation state determines its capacity to maintain CD8(+) T memory precursors for antitumor immunity. Nat. Commun. 8 , 2125 (2017).

21. Pace, L. et al. The epigenetic control of stemness in CD8(+) T cell fate commitment. Science 359, 177-186 (2018).

22. Zhao, E. et al. Cancer mediates effector T cell dysfunction by targeting microRNAs and EZH2 via glycolysis restriction. Nat. Immunol. 17, 95-103 (2016).

23. Alfei, F. et al. TOX reinforces the phenotype and longevity of exhausted T cells in chronic viral infection. Nature 571, 265-269 (2019).

24. Khan, O. et al. TOX transcriptionally and epigenetically programs $\mathrm{CD} 8(+) \mathrm{T}$ cell exhaustion. Nature 571, 211-218 (2019).

25. Scott, A. C. et al. TOX is a critical regulator of tumour-specific $\mathrm{T}$ cell differentiation. Nature 571, 270-274 (2019).

26. Chen, J. et al. NR4A transcription factors limit CAR T cell function in solid tumours. Nature 567, 530-534 (2019).

27. Kao, C. et al. Transcription factor T-bet represses expression of the inhibitory receptor PD-1 and sustains virus-specific $\mathrm{CD} 8+\mathrm{T}$ cell responses during chronic infection. Nat. Immunol. 12, 663-671 (2011).

28. Liu, X. et al. Genome-wide analysis identifies NR4A1 as a key mediator of T cell dysfunction. Nature 567, 525-529 (2019).

29. Seo, H. et al. TOX and TOX2 transcription factors cooperate with NR4A transcription factors to impose CD8(+) T cell exhaustion. Proc. Natl Acad. Sci. USA 116, 12410-12415 (2019).

30. Mohammad, H. P. et al. A DNA hypomethylation signature predicts antitumor activity of LSD1 inhibitors in SCLC. Cancer Cell 28, 57-69 (2015).

31. Sheng, W. et al. LSD1 ablation stimulates anti-tumor immunity and enables checkpoint blockade. Cell 174, 549-563.e519 (2018).

32. Palucka, A. K. \& Coussens, L. M. The basis of oncoimmunology. Cell 164, 1233-1247 (2016).

33. Appay, V., Douek, D. C. \& Price, D. A. CD8+ T cell efficacy in vaccination and disease. Nat. Med. 14, 623-628 (2008).

34. Wei, S. C. et al. Distinct cellular mechanisms underlie anti-CTLA-4 and antiPD-1 checkpoint blockade. Cell 170, 1120-1133 el117 (2017).

35. Carmona, S. J., Siddiqui, I., Bilous, M., Held, W. \& Gfeller, D. Deciphering the transcriptomic landscape of tumor-infiltrating CD8 lymphocytes in B16 melanoma tumors with single-cell RNA-Seq. Oncoimmunology 9, 1737369 (2020).

36. Jadhav, R. R. et al. Epigenetic signature of PD-1+ TCF1+ CD8 T cells that act as resource cells during chronic viral infection and respond to PD-1 blockade. Proc. Natl Acad. Sci. USA 116, 14113-14118 (2019).

37. Majello, B., Gorini, F., Sacca, C. D. \& Amente, S. Expanding the role of the histone lysine-specific demethylase LSD1 in cancer. Cancers (Basel) 11, https://doi.org/10.3390/cancers11030324 (2019).

38. Shi, Y. J. et al. Regulation of LSD1 histone demethylase activity by its associated factors. Mol. Cell 19, 857-864 (2005).

39. Cadigan, K. M. \& Waterman, M. L. TCF/LEFs and Wnt signaling in the nucleus. Cold Spring Harb Perspect Biol 4, https://doi.org/10.1101/ cshperspect.a007906 (2012).

40. Shi, Y. et al. Histone demethylation mediated by the nuclear amine oxidase homolog LSD1. Cell 119, 941-953 (2004).

41. Korinek, V. et al. Constitutive transcriptional activation by a beta-catenin-Tcf complex in APC-/- colon carcinoma. Science 275, 1784-1787 (1997).

42. Gonsalves, F. C. et al. An RNAi-based chemical genetic screen identifies three small-molecule inhibitors of the Wnt/wingless signaling pathway. Proc. Natl Acad. Sci. USA 108, 5954-5963 (2011).

43. Xing, S. et al. Tcf1 and Lef1 transcription factors establish CD8(+) T cell identity through intrinsic HDAC activity. Nat. Immunol. 17, 695-703 (2016).

44. Trapani, J. A. Granzymes: a family of lymphocyte granule serine proteases. Genome Biol. 2, REVIEWS3014 (2001).

45. Schietinger, A. et al. Tumor-specific $\mathrm{T}$ cell dysfunction is a dynamic antigendriven differentiation program initiated early during tumorigenesis. Immunity 45, 389-401 (2016)

46. Henning, A. N., Roychoudhuri, R. \& Restifo, N. P. Epigenetic control of CD8(+) T cell differentiation. Nat. Rev. Immunol. 18, 340-356 (2018).

47. Lee, P. P. et al. A critical role for Dnmt1 and DNA methylation in T cell development, function, and survival. Immunity 15, 763-774 (2001).
48. Gullicksrud, J. A. et al. Differential requirements for Tcf1 long isoforms in $\mathrm{CD} 8(+)$ and $\mathrm{CD} 4(+) \mathrm{T}$ cell responses to acute viral infection. J. Immunol. 199, 911-919 (2017).

49. Chen, Z. et al. TCF-1-centered transcriptional network drives an effector versus exhausted CD8 T cell-fate decision. Immunity 51, 840-855 e845 (2019).

50. Zhao, X. et al. Beta-catenin and gamma-catenin are dispensable for $T$ lymphocytes and AML leukemic stem cells. Elife 9, https://doi.org/10.7554/ eLife.55360 (2020).

51. Gattinoni, L. et al. Wnt signaling arrests effector $\mathrm{T}$ cell differentiation and generates CD8+ memory stem cells. Nat. Med. 15, 808-813 (2009).

52. Jeannet, G. et al. Essential role of the Wnt pathway effector Tcf-1 for the establishment of functional CD8 T cell memory. Proc. Natl Acad. Sci. USA 107, 9777-9782 (2010).

53. Grumolato, L. et al. beta-Catenin-independent activation of TCF1/LEF1 in human hematopoietic tumor cells through interaction with ATF2 transcription factors. PLoS Genet. 9, e1003603 (2013).

54. Lan, F. et al. Recognition of unmethylated histone $\mathrm{H} 3$ lysine 4 links BHC 80 to LSD1-mediated gene repression. Nature 448, 718-722 (2007).

55. Lee, M. G., Wynder, C., Cooch, N. \& Shiekhattar, R. An essential role for CoREST in nucleosomal histone 3 lysine 4 demethylation. Nature 437, 432-435 (2005).

56. Schenk, T. et al. Inhibition of the LSD1 (KDM1A) demethylase reactivates the all-trans-retinoic acid differentiation pathway in acute myeloid leukemia. Nat. Med. 18, 605-611 (2012).

57. Harris, W. J. et al. The histone demethylase KDM1A sustains the oncogenic potential of MLL-AF9 leukemia stem cells. Cancer Cell 21, 473-487 (2012).

58. Zhou, A. et al. Nuclear GSK3beta promotes tumorigenesis by phosphorylating KDM1A and inducing its deubiquitylation by USP22. Nat. Cell Biol. 18, 954-966 (2016).

59. Zhang, X. et al. Pluripotent stem cell protein Sox2 confers sensitivity to LSD1 inhibition in cancer cells. Cell Rep. 5, 445-457 (2013).

60. Kerenyi, M. A. et al. Histone demethylase Lsd1 represses hematopoietic stem and progenitor cell signatures during blood cell maturation. Elife 2, e00633 (2013).

61. Laurent, B. et al. A specific LSD1/KDM1A isoform regulates neuronal differentiation through H3K9 demethylation. Mol. Cell 57, 957-970 (2015).

62. Christopher, M. A. et al. LSD1 protects against hippocampal and cortical neurodegeneration. Nat. Commun. 8, 805 (2017).

63. Wang, J. et al. LSD1n is an H4K20 demethylase regulating memory formation via transcriptional elongation control. Nat. Neurosci. 18, 1256-1264 (2015).

64. Fang, Y., Liao, G. \& Yu, B. LSD1/KDM1A inhibitors in clinical trials: advances and prospects. J. Hematol. Oncol. 12, 129 (2019).

65. Dobin, A. et al. STAR: ultrafast universal RNA-seq aligner. Bioinformatics 29, 15-21 (2013).

66. Frankish, A. et al. GENCODE reference annotation for the human and mouse genomes. Nucleic Acids Res. 47, D766-D773 (2019).

67. Liao, Y., Smyth, G. K. \& Shi, W. featureCounts: an efficient general purpose program for assigning sequence reads to genomic features. Bioinformatics $\mathbf{3 0}$ 923-930 (2014).

68. Love, M. I., Huber, W. \& Anders, S. Moderated estimation of fold change and dispersion for RNA-seq data with DESeq2. Genome Biol. 15, 550 (2014).

69. Hansen, K. D., Irizarry, R. A. \& Wu, Z. Removing technical variability in RNA-seq data using conditional quantile normalization. Biostatistics 13, 204-216 (2012).

70. Subramanian, A. et al. Gene set enrichment analysis: a knowledge-based approach for interpreting genome-wide expression profiles. Proc. Natl Acad. Sci. USA 102, 15545-15550 (2005).

\section{Acknowledgements}

We thank Drs. Phil Greenberg, Jianzhu Chen, and Xi He for critical reading of the manuscript and valuable suggestions. We thank Dr. Stuart Orkin for sharing Lsd 1 floxed mouse strain, Dr. Hai-Hui Xue for sharing Tcf7 plasmids and Dr. Xi He for sharing reagents related to the TCF/LEF reporter assay and providing advices on experiments. We thank Drs. Arlene Sharpe, Nick Haining, Anna Anderson, David Fisher, and Glenn Dranoff for sharing mouse cell lines. We thank all the Shi lab members for discussions and suggestions. We thank NIH Tetramer Core Facility for sharing SIINFEKL $\mathrm{H}-2 \mathrm{~Kb}$ Tetramer. This work was supported by the Outstanding Investigator Award from the NCI (R35 CA210104), as well as funds from Boston Children's Hospital and Ludwig Institute for Cancer Research to Y.S. Y.S. is an American Cancer Society Research Professor. The work by M.L. was performed in the laboratory of Nenad Sestan and supported by NIH grants P50 MH106934 and U01 MH116488.

\section{Author contributions}

W.S. and Y.S. conceived and initiated the project; Y. L. and W.S. designed and performed all experiments with help from B.D. and Z.S.; M.L. performed bioinformatic analysis of the RNAseq data; Y.L., W.S. and Y.S. wrote the manuscript with input from B.D., M.L. and Z.S. 


\section{Competing interests}

Y.S. is a co-founder and equity holder of K36 Therapeutics, a consultant for Active Motif, and holds equity in Imago Biosciences. Y.S. is a member of the Scientific Advisory Board of the College of Life Sciences, West Lake University, and a member of the MD Anderson External Advisory Board. All other authors declare no competing interests.

\section{Additional information}

Supplementary information The online version contains supplementary material available at https://doi.org/10.1038/s41467-021-27179-7.

Correspondence and requests for materials should be addressed to Wanqiang Sheng or Yang Shi.

Peer review information Nature Communications thanks the anonymous reviewer(s) for their contribution to the peer review of this work.

Reprints and permission information is available at http://www.nature.com/reprints
Publisher's note Springer Nature remains neutral with regard to jurisdictional claims in published maps and institutional affiliations.

\section{(c) (i)}

Open Access This article is licensed under a Creative Commons Attribution 4.0 International License, which permits use, sharing, adaptation, distribution and reproduction in any medium or format, as long as you give appropriate credit to the original author(s) and the source, provide a link to the Creative Commons license, and indicate if changes were made. The images or other third party material in this article are included in the article's Creative Commons license, unless indicated otherwise in a credit line to the material. If material is not included in the article's Creative Commons license and your intended use is not permitted by statutory regulation or exceeds the permitted use, you will need to obtain permission directly from the copyright holder. To view a copy of this license, visit http://creativecommons.org/ licenses/by/4.0/.

(c) The Author(s) 2021, corrected publication 2022 